\title{
TABLES TO FACILITATE THE COMPUTATION OF THE PROBABLE ERRORS OF THE CHIEF CONSTANTS OF SKEW FREQUENCY DISTRIBUTIONS.
}

\author{
By A. RHIND. B.Sc., Biometric Laboratory, University College, London.
}

Tas general theory of the probable errors of the constants of skew frequency distributions was originally given with illustrations by Pearson and Filon, Phil. Trans. Vol. 191, A (1898), pp. 229-311. The values there deduced depend on the form of special frequency curve adopted, and involve considerable arithmetical work for each individual case. In these frequency investigations the fundamental constants are the well-known $\beta_{1}$ and $\beta_{9}$. Every frequency character expressible in terms of $\beta_{1}$ and $\beta_{2}$ can have its probable error determined, provided we know the probable errors of $\beta_{1}$ and $\beta_{2}$ and the correlation in deviations between $\beta_{1}$ and $\beta_{2}$. General expressions for the probable errors (or the S.D.'s $\Sigma_{\beta_{1}}, \Sigma_{\beta_{1}}$ ) of $\beta_{1}$ and $\beta_{8}$ as well as the correlation $R_{\beta_{1} A_{2}}$ of deviatious in $\beta_{1}$ and $\beta_{1}$, together with the probable error of the criterion (or its S.D. $=\Sigma_{x}$ ) were first given by Pearson, Phil. Trans. Vol. 198, A (1902). These involve a knowledge of $\beta_{8}, \beta_{5}, \beta_{4}$ and $\beta_{3} *$, further constants of the distribution which can only be found if the numerical values of $\mu_{3}, \mu_{0}, \mu_{3}$ and $\mu_{0}$ have been in some way determined. Now it has been shown that with the total frequencies usual in practice these bigh moments are subject to very large percentage errorst, rendering their use extremely undesirable, even if we could overcome our natural repugnance to the great labour of calculating them.

But we bave to bear in mind that the exact value of a probable error is not usually desired. What we more often require is a rough determination of its

- $\beta_{3}=\mu_{3} \mu_{8} / \mu_{2} 4, \quad \beta_{4}=\mu_{0} / \mu_{3}^{3}, \quad \beta_{6}=\mu_{7} \mu_{3} / \mu_{3} \beta_{1}, \beta_{6}=\mu_{0} / \mu_{2}^{4}$.

+ Pearcon: "On the General Theory of gkew Corralation and Non-Linear Regression," Drapers" Company Researeh Dlemoirs (Dulad \& Co., 1905), p. 8. 
magnitude in order that we may appreciate whether a certain quantity is or is not really significant. Accordingly it had been the practice of the Biometric Laboratory, if a frequency was only moderately a-normal, to use the values of the higher $\beta$ 's in determining probable errors, which would flow from the assumption of normality; if on the uther hand the distribution had values of $\beta_{1}$ and $\beta_{2}-3$ differing a good deal from zero, to assume that the higher moments might be obtained from a skew distribution of Type III. (i.e. a distribution for which $2 \beta_{2}-3 \beta_{1}-6=0$ ). The justification of such hypotheses lies in the fact that if our data are to be of much value, the probable errors must themselves be small, hence in calculating these errors it is legitimate to insert into the formulae for them values for the $\beta$ 's that only differ from their true values by small quantities. Such insertion can only introduce second order, and therefore for our purposes usually unimportant, changes in the probable error. This point is emphasised when we remember the large percentage errors of the high $\beta^{\prime} \mathrm{s}$. For example, it is usual to take the probable error of a standard deviation $\sigma=67449 \sigma / \sqrt{2 n}$, but its true value $=\cdot 67449 \frac{\sigma}{\sqrt{2 n}}\left(1+\frac{1}{2} \eta\right)^{\frac{1}{2}}$; the forner really results from assuming the kurtosis, $\eta=\beta_{2}-3$, to have the value zero of the normal curve. There are very few arguments made from probable error which would be seriously affected if the probable error were altered by 25 per cent. of its value, or if $\eta$ took values from -.875 to $1 \cdot 125$, i.e. we might give $\beta_{2}$ any value between $2 \cdot 125$ and $4 \cdot 125$ to get in practice a sufficiently close result.

Now the object of the present paper is to extend this idea by applying a method of determining $\beta_{3}, \beta_{4}, \beta_{8}$ and $\beta_{8}$ still more exact than the methods indicated above. It is well-known that the frequency curves in common use are deduced from the integral of

$$
\frac{1}{y} \frac{d y}{d x}=\frac{a x+b}{c_{0}+c_{2} x+c_{2} x^{2}}
$$

and that this really assumes the condition that the coefficients of higher terms in the denominator on the right, e.g. $c_{3}, c_{4}$, etc, are all zero. These conditions involve a finite difference relation between the successive moments, first published in $1903^{*}$, and enable us to determine any higher moment from the first three $\mu_{2}, \mu_{2}$ and $\mu_{4}$. Such a finite difference momental equation actually exists for all probability frequency distributions of the hypergeometrical series type, which cover so wide a range of chance problems.

It is practically impossible to determine in a large percentage of cases whether the higher moments do or do not within their probsble errors obey this finite difference relation, for the reason above stated, i.e. the bigh values of their probable errors. The present tables assume that they do; in other words $\beta_{3}, \beta_{4}$, 
$\beta_{1}$ and $\beta_{8}$ are calculated from $\beta_{1}$ and. $\beta_{2}$, on the assumption that the values of them obtained from the finite difference momental formuls are sufficiently accurate to use in Pearson's formulae for $\Sigma_{p_{1}}, \Sigma_{p_{1}}, R_{p_{1} \beta_{1}}$ and $\Sigma_{c_{k}}$, etc.

The following are the finite difference $\beta$-formulse used :

$$
\begin{aligned}
& \beta_{n} \text { (even) }=(n+1)\left\{\frac{\beta_{n-1}}{2}+\left(i+\frac{a}{2}\right) \beta_{n-1}\right\} /\left(1-\frac{n-1}{2} \alpha .\right), \\
& \beta_{n} \text { (odd) }=(n+1)\left\{\frac{\beta_{1}}{2} \beta_{n-1}+\left(1+\frac{a}{2}\right) \beta_{n-3}\right\} /\left(1-\frac{n-1}{2} \alpha\right),
\end{aligned}
$$

where $a=\left(2 \beta_{2}-3 \beta_{1}-6\right) /\left(\beta_{2}-3\right)$.

The process of calculation adopted was as follows:

Fundamental values of $\beta_{1}, \beta_{2}$. were adopted; these are indicated in Table VI, and the resulting values of $\beta_{3}, \beta_{4}, \beta_{5}, \beta_{6}$ calculated by the above formula were then found to seven figurea. These are tabulated to six figures as they may be of service for the determinstion of other constants as occasion arises.

The values of $\mu_{4}$ for the different values of $\beta_{1}$ and $\beta_{2}$ being known, it was seen that a very simple diagram would permit of a statistician ascertaining at once from his values of $\beta_{2}$ and $\beta_{2}$ the type of his frequency distribution. In fact this diagram brings out very suggestively the normal curve "point" (G), the Types II., III., V. and VII." "lines" and the Types I., IV. and VI. "areas" of cccurrence. By aid of this diagram and a reasonable consideration of the probable errors of his $\beta_{1}$ and $\beta_{2}$ the statistician can readily determine wiuhin what limits be is justified in using any special type of frequency curve for given data.

The following values of anslytic constants for the fundamental values were then found $\sqrt{N} \Sigma_{p_{1}}$ (Table I), $\sqrt{N} \Sigma_{p_{1}}$ (Table II), $R_{p_{1} \beta_{1}}$ (Table III.), and intermediate values deduced by interpolatiun. It is believed that these interpolated values recorded to two places of decimals only are amply sufficient for the practical uses to which these tables will be put.

From these tables were found, again using interpolation, the probable errors of the two chief desired physical constants: (i) $d$, the distance from mean to mode or the modal divergence, and (ii) $S k$, the skewness of the distribution. In Table IV. we have the values of $\sqrt{N} \Sigma_{d} / \sigma$ and in Table $V$. the values of $\sqrt{N} \Sigma_{a t}$ provided. The actual quantities tabled in Tables I., II, IV. and V. are such that when multiplied by $67449 / \sqrt{N}$ we obtain immediately the required probable exrors. The value of this numerical factor can at once, however, be extracted from Winifred Gibeon's Tables for computing probsble errorst.

- It is convenient to oall $y=y_{0}\left(1+\frac{z^{2}}{a^{3}}\right)^{-}$Type VII., 200 Biometrika, Vol. Tr., p. 174.

+ Biometrika, Vol. IV., p. 385 .

Biometriks vx 
In the actual construction of the tables fundamental valnes of $\beta_{1}$ and $\beta_{2}$ were taken covering the usual range of these quantities in actual practice. The tables are, however, limited by certain considerations, which are not without suggestiveness for theoretical frequency discussions Along a certain line, indicated in our diagram, $\beta_{6}$ becomes infinite. But this is statistically impossible. Hence, either we never get frequency distributions having such values of $\beta_{1}$ and $\beta_{2}$, or if we do the finite difference moment formula cannot approximate in such cases to the true state of affairs, and we must introduce an additional coefficient $c_{3}$ into the denominator of the right-hand side of the fundamental differential equation. The discussion and classification of such curves are now in hand. On the diagram the areas where they are absolutely needful are marked Heterotypic.

It is believed that the diagrams and tables now published will prove extremely useful to the biometrician. Having determined his $\beta_{1}$ and $\beta_{8}$, he will be able to see at a glance whether his frequency distribution may be safely treated by the usual types or is heterotypic. If it be one of the usual types, he will know at once how to classify it. Next an examination of Tables I., II., III and IV., or such examination with a short interpolation, will give him with sufficient practical accuracy the probable errors of $\beta_{1}, \beta_{2}$, the modal divergence $(d)$ and the skewness $(S k)$. Should other and more elaborate probable errors be required, they will be deducible from Tables I., II. and III., or by using Table VI., for all the usually desired constants depend upon the quantities therein tabulated.

The two diagrams represent, (A), a practical working diagram covering the customary range of $\beta_{1}$ and $\beta_{2}$ and, (B), a diagram on a small scale sbowing the whole nature of the distribution of the type curves from the theoretical standpoint.

The upper part of either diagram is bounded by the line $4 \beta_{2}-3 \beta_{1}=0$, because it has been shown that $\beta_{2}$ is of necessity $>\beta_{1}^{*}$. The normal curve is represented by a point $\beta_{1}=0, \beta_{2}=3$ marked $G$ on the diagran.

The transition Types $\mathrm{WI}$. and V. are given by values of $\beta_{1}, \beta_{2}$ on the line

and on the cubic

$$
2 \beta_{2}-3 \beta_{1}-6=0 \text {, }
$$

respectively.

$$
\beta_{1}\left(\beta_{2}+3\right)^{9}=4\left(4 \beta_{2}-3 \beta_{1}\right)\left(2 \beta_{2}-3 \beta_{1}-6\right),
$$

Type II. is a particular case of Type I. for $\beta_{1}=0$, and Type VII a particular case of 'Type IV. for $\beta_{1}=0$; they are thus represented by portions of the vertical running through the "nornial point" $G$. While the upper boundary of the diagram lies in the $U$-curve part of Type $I$, to be discussed below, the lower boundary is fixed by the line $8 \beta_{2}-15 \beta_{1}-36=0$ along which the finite difference moment formula first fails. This matter needs a little consideration.

\footnotetext{
- Seo Perrson : Phil. Trans. Vol. 186, A, pp. 343-414.
} 
Diagrax a.

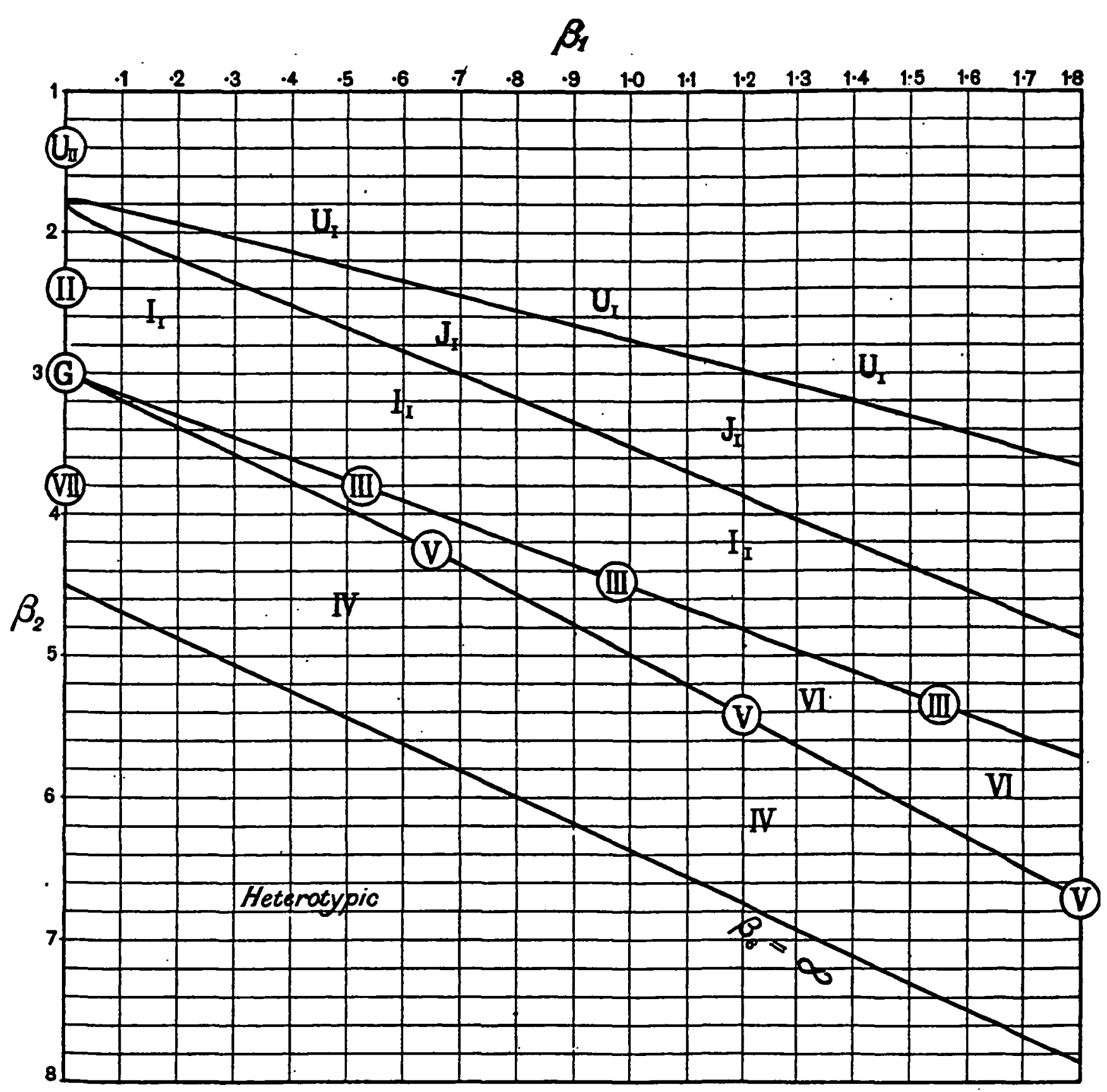


- Dingrax B.

$\beta_{1}$

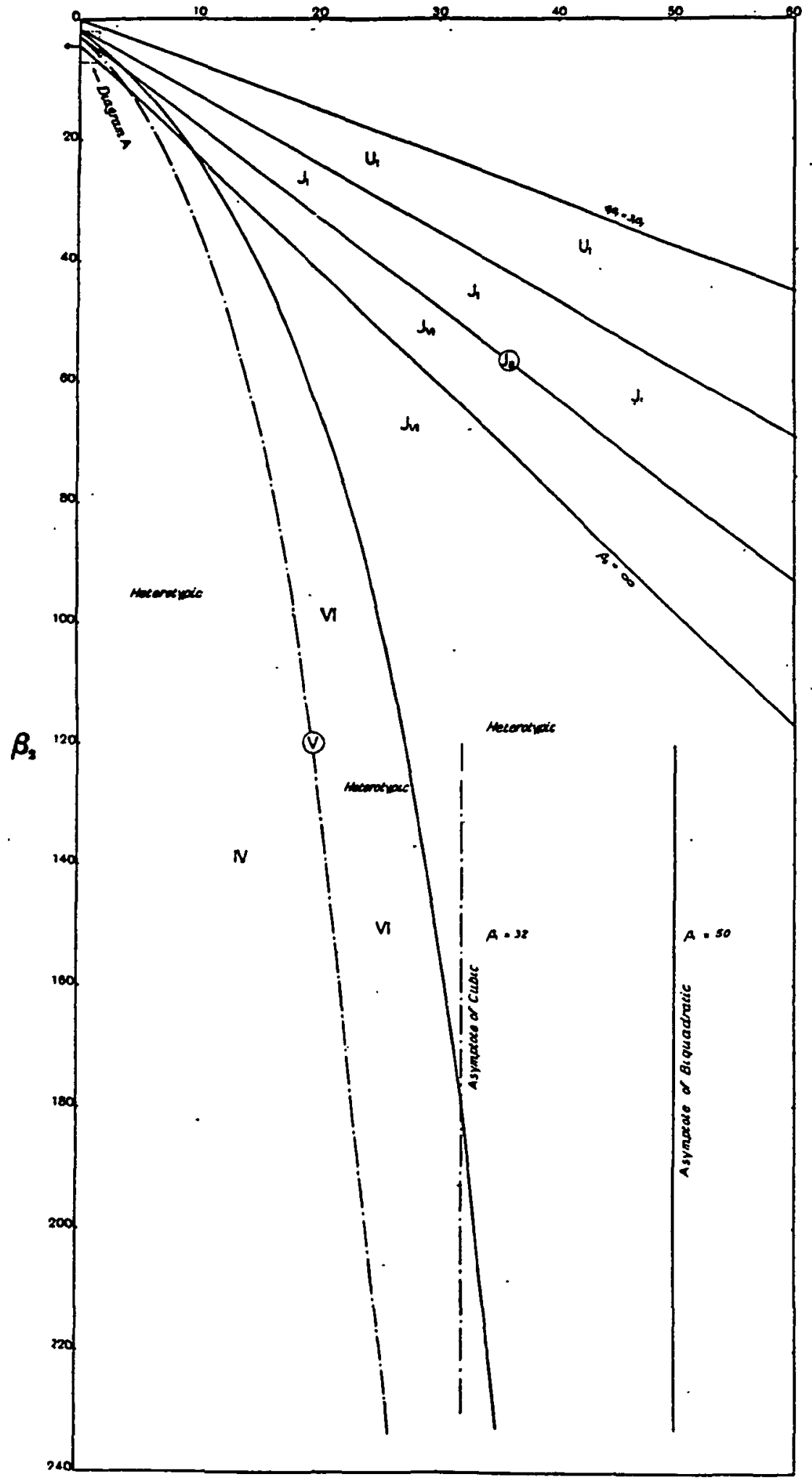


The equation to Type IV. is

$$
y=y_{0}\left\{1+\frac{x^{2}}{a^{2}}\right\}^{-\frac{1}{2}(r+2)} e^{->\tan ^{-1}(x / a)},
$$

with the reduction formula *

$$
\mu_{n+1}=\frac{n}{r-n}\left(\frac{a^{2} \mu_{n-1}}{\cos ^{2} \phi}-2 a \tan \phi \mu_{n}\right)
$$

where $\tan \phi=\nu / r$ and $r=6\left(\beta_{2}-\beta_{1}-1\right) /\left(2 \beta_{2}-3 \beta_{1}-6\right)$. It is clear that when $r=n, \mu_{n+1}$ becomes infinite. Now the probable errors of $\beta_{1}$ and $\beta_{2}$ require us to go as far as $\mu_{0}$. Hence $r$ must be greater than 7 if we are to use this formula, and this gives us at once the limiting line

$$
8 \beta_{2}-15 \beta_{1}-36=0 \text {. }
$$

Of course the difference formuls would also fail for $\mu_{3}, \mu_{8}$ or $\mu_{4}$, if $r$ were equal to 4,5 or 6 . But all the resulting lines lie outside the above line, which is all we need take into account. The failure of the difference formula is easily seen if we remember that

$$
\frac{1}{y} \frac{d y}{d x}=\frac{a_{0}+a_{2} x}{c_{0}+c_{2} x+c_{3} x^{2}}
$$

leads at once for this type to

$$
\begin{gathered}
\int_{-\infty}^{+\infty} x^{n}\left(a_{0}+a_{1} x\right) y d x=\int_{-\infty}^{+\infty} \cdot\left(c_{0}+c_{1} x+c_{2} x^{n}\right) x^{n} d y \\
N\left(a_{0} \mu_{n}+a_{1} \mu_{n+1}\right)=-N\left(c_{0} n \mu_{n-1}+c_{1}(n+1) \mu_{n}+c_{2}(n+2) \mu_{n+1}\right) \\
+\left[y\left(c_{0} x^{n}+c_{1} x^{n+1}+c_{2} x^{n+2}\right)\right]_{-\infty}^{+\infty} .
\end{gathered}
$$

or

The difference formuls above follows from supposing the term between brackets to vanish at the limits.

But this it will not do unless

$$
\left[\frac{x^{n+2}}{\left(a^{2}+x^{2}\right)^{\frac{1}{2(r+2)}}}\right]_{x=\infty}=0
$$

or if $a$ be finite, unless $r$ be $>n$, or to apply present results $r>7$.

Of course for any real data $\mu_{3}$ may become large, but it cannot actually become infinite. Fairly good fits-owing to the agreement of the first four moments-msy be found even near the line $8 \beta_{2}-15 \beta_{1}-36=0$, but if we want to get the probable errors of $\beta_{1}$ and $\beta_{2}$ in this neighbourhood, it is best to calculate the higher moments and $\beta_{3}, \beta_{6}, \beta_{6}$ and $\beta_{6}$ from the actual data. Outoide this line 
we have marked the area as Heterotypic, because theoretically we need to introduce further terms into the denominator of our expression, i.e. to use $c_{3}$.

We now pass to a consideration of the subtypes of Type I. The equation is *

$$
y=y_{0}\left(1+\frac{x}{a_{1}}\right)^{m_{1}}\left(1-\frac{x}{a_{2}}\right)^{m_{4}},
$$

where $m_{1}$ and $m_{1}$ are roots of the quadratic

and

$$
m^{2}-m(r-2)+e-r+1=0
$$

$$
r=6\left(\beta_{2}-\beta_{1}-1\right) /\left(3 \beta_{1}-2 \beta_{2}+6\right) \text {, }
$$

$$
e=\frac{r^{2}}{4+1 \beta_{1}(r+2)^{2} /(r+1)} .
$$

Now $m_{1}$ and $m_{2}$ will either be both positive or both negative if $e-r+1$ is positive, or the curve $\epsilon-r+1=0$ separates the area of $J$-curves or.modeless curves from the area of modal curves $\left(I_{1}\right.$ curves) and the area of anti-modal curves or $U$-curves.

$\epsilon-r+1=0$ is the biquadratic

$$
\beta_{1}\left(8 \beta_{2}-9 \beta_{1}-12\right) /\left(4 \beta_{1}-3 \beta_{1}\right)=\left(10 \beta_{2}-12 \beta_{1}-18\right)^{2} /\left(\beta_{2}+3\right)^{2} .
$$

This biquadratic was traced by expressing it in the form:

$$
\beta_{1}=4(1+2 \alpha)^{2}(2+\alpha) /(2+3 \alpha),
$$

and then finding $\beta_{2}$ for a series of values of $\alpha$ and determining $\beta_{3}$ from the equation

$$
a=\left(2 \beta_{2}-3 \beta_{1}-6\right) /\left(\beta_{2}+3\right) \text {. }
$$

Within the loop of the biquadratic all curves are $J$-curves, and the term "skewness" loses its essential meaning. Within this area, it will be noticed, our tables do not give the probable error of the skewness or of the modal divergence. Above this loop and up to the line $4 \beta_{2}-3 \beta_{1}=0$, we are in the range of $U$-curves and the skewness signifies the ratio to the standard deviation of the distance from mean to anti-mode. Below the loop we are in the customary Type I. area with $m_{1}$ and $m_{3}$ both positive.

Our second Diagram, B, shows what becomes of the biquadratic limiting the $J$-shaped curves. It first meets the Type III. line, and at this point Type III. curves become $J$-curvest and cease to have a true mode distinct from the asymptote value. The biquadratic then passes into the Type VI. area and Type VI. curves become $J$-curves beyond this. It never crosses, however, into

- Pearson: Phil. Trans. Vol. 186, A, pp. 367-371.

+ Pearson : Phil. Trans. Vol. 186, A, p. 374 and Plnte 0, Fig. 5. 
Type IV. area, for the cubic which bounds Type IV. asymptotes to the vertical line $\beta_{1}=32$, and the biquadratic asymptotes to the vertical line $\beta_{1}=50$ as shown on the diagram.

Of course much of Diagram $B$ extends bejond the values of $\beta_{1}$ and $\beta_{2}$ that we are familiar with in actual frequency distributions. For practical purposes Diagram A drawn to a much larger scale suffices, and guides the statistician to the appropriate type and to the probable errors Nevertheless Diagram B will indicate many points of much thenretical interest, and serves to show where failure in curve fitting is likely to arise. 
TABLE I.

Values of $\sqrt{\bar{N}} \Sigma_{\beta_{1} \text { : }}$

$\beta_{1}$

\begin{tabular}{|c|c|c|c|c|c|c|c|c|c|c|c|c|c|c|c|c|}
\hline & 0.00 & 0.05 & 0.10 & $0 \cdot 15$ & 0.20 & 0.25 & 0.30 & 0.35 & 0.40 & 0.45 & 0.50 & 0.55 & .0 .60 & 0.65 & 0.70 & 0.75 \\
\hline $2 \cdot 0$ & $\infty$ & •58 & 93 & $1 \cdot 15^{\circ}$ & $1 \cdot 37$ & 1.57 & $1 \cdot 77$ & 1.07 & $2 \cdot 17$ & $2 \cdot 38$ & $2 \cdot 58$ & 2.80 & 302 & $3 \cdot 24$ & 3.46 & 3.71 \\
\hline & 00 & 0.59 & & $1 \cdot 12$ & $1 \cdot 30$ & 1.50 & $1 \cdot 70$ & 1.90 & $2 \cdot 10$ & $2 \cdot 29$ & $2 \cdot 48$ & 2.69 & 2.01 & $3 \cdot 18$ & $3 \cdot 34$ & 3.57 \\
\hline & .00 & 0.60 & 97 & $1 \cdot 13$ & $1 \cdot 30$ & $i \cdot 48$ & 1.67 & 1.86 & & & & $2 \cdot 61$ & & & & $3 \cdot 44$ \\
\hline & & 0.62 & & $1 \cdot 15$ & $1 \cdot 32$ & 1.48 & 16 & $1 \cdot 84$ & $2 \cdot 02$ & $2 \cdot 19$ & & $2 \cdot 35$ & & & $3 \cdot 12$ & $3 \cdot 32$ \\
\hline & 0.00 & 0.64 & 1 & 1.20 & $1 \cdot 37$ & 1.54 & $1 \cdot 70$ & 1.86 & 2.02 & $2 \cdot 18$ & $2 \cdot 34$ & 2.51 & $2 \cdot 68$ & $2 \cdot 85$ & 303 & $3 \cdot 22$ \\
\hline 2.5 & 0.00 & 0.66 & 105 & 1.26 & $1 \cdot 45$ & 161 & $1 \cdot 76$ & 1.91 & $2 \cdot 05$ & $2 \cdot 19$ & $2 \cdot 33$ & $2 \cdot 49$ & $2 \cdot 65$ & $2 \cdot 81$ & $2 \cdot 97$ & $3 \cdot 14$ \\
\hline 26 & 0.00 & 0.69 & $1 \cdot 10$ & 1.34 & 1.54 & $1 \cdot 72$ & 1.86 & $2 \cdot 00$ & $2 \cdot 13$ & & $2:$ & $2 \cdot 30$ & $2 \cdot 64$ & $2 \cdot 78$ & $2 \cdot 92$ & 3.08 \\
\hline $2 \cdot 7$ & 0.00 & 0.73 & $1 \cdot 15$ & & & & 1.96 & 2.09 & & $2 \cdot 32$ & $2 \cdot 42$ & $2 \cdot 53$ & $2 \cdot 65$ & $2 \cdot 77$ & $2 \cdot 90$ & 3.05 \\
\hline $2 \cdot 8$ & 0.00 & 0.77 & 2.22 & 1.51 & & 1.94 & 2.07 & $2 \cdot 20$ & $2 \cdot 32$ & $2 \cdot 41$ & $2 \cdot 50$ & $2 \cdot 60$ & $2 \cdot 70$ & 80 & 2-93 & 3.05 \\
\hline $2 \cdot 9$ & 0.00 & 0.81 & & $1 \cdot 61$ & 1.8 & & $\mathbf{2} \cdot 20$ & $2 \cdot 33$ & $\mathbf{z} \cdot 44$ & $\mathbf{2} \cdot \mathbf{3 3}$ & $2 \cdot 62$ & $2 \cdot 70$ & $z \cdot 79$ & $2 \cdot 89$ & $2 \cdot 99$ & 309 \\
\hline$s .0$ & 0.00 & 0.87 & $1 \cdot 40$ & $1 \cdot 73$ & 201 & $\mathbf{2} \cdot 20$ & $2 \cdot 34$ & $2 \cdot 47$ & $8 \cdot 57$ & $2 \cdot 67$ & $2 \cdot 76$ & $2 \cdot 84$ & $2 \cdot 92$ & 3.00 & 3.09 & $3 \cdot 18$ \\
\hline & 0.00 & 0.94 & 1.53 & $1 \cdot 86$ & $2 \cdot 17$ & $2 \cdot 35$ & $2 \cdot 51$ & $2 \cdot 64$ & $2 \cdot 75$ & 2.85 & 2.04 & 3.00 & 3.08 & $3 \cdot 15$ & $3 \cdot 23$ & $3 \cdot 30$ \\
\hline$s$ & 0.00 & 1.02 & 1.67 & $\mathbf{z} 0 \mathrm{z}$ & $2 \cdot 33$ & $2 \cdot 52$ & $2 \cdot 71$ & $2 \cdot 84$ & $2 \cdot 95$ & 3.05 & $3 \cdot 14$ & $3 \cdot 22$ & $3 \cdot 27$ & $3 \cdot 33$ & $3 \cdot 40$ & 3.46 \\
\hline 3.5 & 0.00 & $1 \cdot 12$ & 82 & & $2 \cdot 50$ & $2 \cdot 71$ & 2.92 & 306 & & $3 \cdot 28$ & $3 \cdot 37$ & 3.44 & 3.50 & & 3.60 & 3.65 \\
\hline & 0.00 & $1 \cdot 24$ & & & & $z \cdot 93$ & $3 \cdot 14$ & $3 \cdot 30$ & & & 3.63 & & & 3. & & \\
\hline & 0.0 & 137 & & $2 \cdot 57$ & & $3 \cdot 17$ & 3.39 & & & & 3.91 & 3.98 & & 406 & $4 \cdot 10$ & $4 \cdot 12$ \\
\hline$s \cdot$ & 0.00 & 1.50 & & & & $3 \cdot 43$ & 36 & 3.84 & $3 \cdot 99$ & $4 \cdot 12$ & $4: 22$ & $4 \cdot 29$ & $4 \cdot 33$ & $4 \cdot 37$ & $4 \cdot 40$ & $4 \cdot 41$ \\
\hline s. & 0.00 & 1.64 & $2 \cdot 50$ & $2 \cdot 99$ & & $3 \cdot 70$ & 3.93 & $4 \cdot 14$ & $4 \cdot 31$ & $4 \cdot 44$ & $4 \cdot 54$ & $4 \cdot 61$ & $4 \cdot 66$ & $4 \cdot 70$ & $4 \cdot 72$ & 4.74 \\
\hline 3. & 0.00 & 1.78 & $2 \cdot 67$ & $3 \cdot 20$ & & 3.97 & $4 \cdot 23$ & $4 \cdot 46$ & $4 \cdot 64$ & $4 \cdot 77$ & 4.87 & 4.95 & 5.00 & 505 & 507 & \\
\hline$s \cdot 9$ & 0.00 & 1.93 & $2 \cdot 86$ & $3 \cdot 43$ & 3.89 & $4 \cdot 20$ & $4 \cdot 54$ & $4 \cdot 79$ & 4.97 & $5 \cdot 11$ & b.23 & $5 \cdot 32$ & $5 \cdot 38$ & $5 \cdot 43$ & $5 \cdot 46$ & 5.48 \\
\hline $4 \cdot 0$ & 0.00 & $2 \cdot 10$ & 3.07 & 3.69 & $4 \cdot 17$ & $4: 56$ & $4: 87$ & $5 \cdot 13$ & 3.32 & $5 \cdot 48$ & $5 \cdot 62$ & 5.72 & $5 \cdot 79$ & 5.84 & 5.88 & 5.89 \\
\hline $4 \cdot 1$ & -1 & - & $3 \cdot 4$ & 3.87 & $4 \cdot 47$ & $4 \cdot 87$ & 5.21 & B.49 & 5.69 & 5.87 & 6.03 & $6 \cdot 15$ & 6.23 & 6.28 & $6 \cdot 32$ & $6 \cdot 33$ \\
\hline $4 \cdot 2$ & - & - & $3 \cdot 53$ & $4 \cdot 19$ & $4 \cdot 79$ & B.21 & 3.58 & $5 \cdot 88$ & $6 \cdot 10$ & $6 \cdot 30$ & 6.46 & 6.60 & 6.69 & 6.75 & 6.80 & 6.81 \\
\hline $4 \cdot 5$ & - & - & $3 \cdot 78$ & $4 \cdot 52$ & $5 \cdot 13$ & 5.68 & 5.97 & $6 \cdot 29$ & 6.54 & 6.75 & 6.93 & $7 \cdot 07$ & $7 \cdot 18$ & $7 \cdot 25$ & $7 \cdot 29$ & $7 \cdot 31$ \\
\hline & & - & & & & $5 \cdot 98$ & & $6 \cdot 74$ & & & $7 \cdot 42$ & $7 \cdot 57$ & $7 \cdot 68$ & $7 \cdot 76$ & 10 & $7 \cdot 83$ \\
\hline & - & 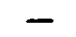 & & 5. & 5.88 & 6.42 & & & & & $7 \cdot 95$ & $8 \cdot 10$ & $8 \cdot 21$ & $8 \cdot 29$ & & \\
\hline & - & - & - & - & - & - & & & & & $8 \cdot 51$ & & & $8 \cdot 85$ & & \\
\hline & - & - & - & - & - & - & & & $8 \cdot 64$ & $8 \cdot 90$ & $9 \cdot 11$ & & $8-35$ & $9 \cdot 44$ & $9 \cdot 50$ & $9 \cdot 54$ \\
\hline 4. & - & - & - & - & - & - & & $8 \cdot 88$ & $9 \cdot 24$ & 9.54 & $9 \cdot 75$ & $9 \cdot 89$ & 8.99 & 10.08 & $10 \cdot 14$ & $10 \cdot 18$ \\
\hline 4 & - & - & - & - & - & - & 903 & $9 \cdot 47$ & $9 \cdot 86$ & $10 \cdot 21$ & $10 \cdot 42$ & 10.58 & $10 \cdot 69$ & $10 \cdot 78$ & 10.84 & $10 \cdot 80$ \\
\hline & & - & - & - & - & - & $9 \cdot 66$ & 10.08 & 10.50 & $10 \cdot 90$ & $11 \cdot 19$ & $11 \cdot 33$ & 1.44 & $11 \cdot 53$ & $11 \cdot 60$ & $11 \cdot 64$ \\
\hline & - & - & - & - & - & - & - & - & - & - & - & - & $12 \cdot 26$ & $12 \cdot 36$ & $12 \cdot 42$ & 12. \\
\hline & - & - & - & - & - & - & - & - & - & & & - & $13 \cdot 10$ & $13 \cdot 26$ & 13 & 13.8 \\
\hline & & - & - & - & & - & - & & & & & & & & $14 \cdot 18$ & $14 \cdot 18$ \\
\hline & - & - & - & - & & - & - & & & & & & & & & \\
\hline & - & - & - & - & & - & - & & & - & & & $1.5 \cdot 90$ & $15 \cdot 98$ & 16.05 & 16.07 \\
\hline & - & - & - & - & - & - & - & - & - & - & - & - & - & - & - & - \\
\hline & & - & - & & & - & $\pi$ & & & - & & 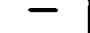 & - & - & & \\
\hline 5. & ב & Z & Z & - & $=$ & E & $\overline{-}$ & 二 & 二 & - & $\bar{z}$ & $\overline{-}$ & - & 7 & & \\
\hline & $=$ & - & 二 & E & - & 二 & $\bar{Z}$ & 二 & 二 & $=$ & $=$ & $=$ & Z & $=$ & $=$ & $=$ \\
\hline & - & - & - & - & - & - & - & - & - & - & - & - & - & - & - & - \\
\hline & - & - & & & & - & - & & & & & & & & & \\
\hline & - & - & & & & & & & & & & & & & & \\
\hline & & & & & & & & & & & & & & & & \\
\hline & & & & & & & & & & - & - & 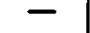 & & & & \\
\hline & & - & & & & 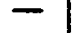 & - & - & - & - & & - & - & & & \\
\hline & & - & & - & & - & - & - & & & & & - & - & - & - \\
\hline 6.8 & - & 二 & $\bar{z}$ & $\bar{z}$ & $\bar{z}$ & $\bar{z}$ & Z & - & 二 & & 二 & & $\bar{E}$ & E & $\bar{z}$ & Z \\
\hline 7.0 & Z & 二 & $\bar{z}$ & $=$ & 二 & $=$ & $\bar{z}$ & $=$ & $\bar{z}$ & $\bar{z}$ & $\bar{z}$ & - & - & $=$ & $=$ & - \\
\hline
\end{tabular}


TABLE I.-(continued).

Values of $\sqrt{N} \Sigma_{\beta_{1}}$

$\boldsymbol{\beta}_{\mathbf{1}}$

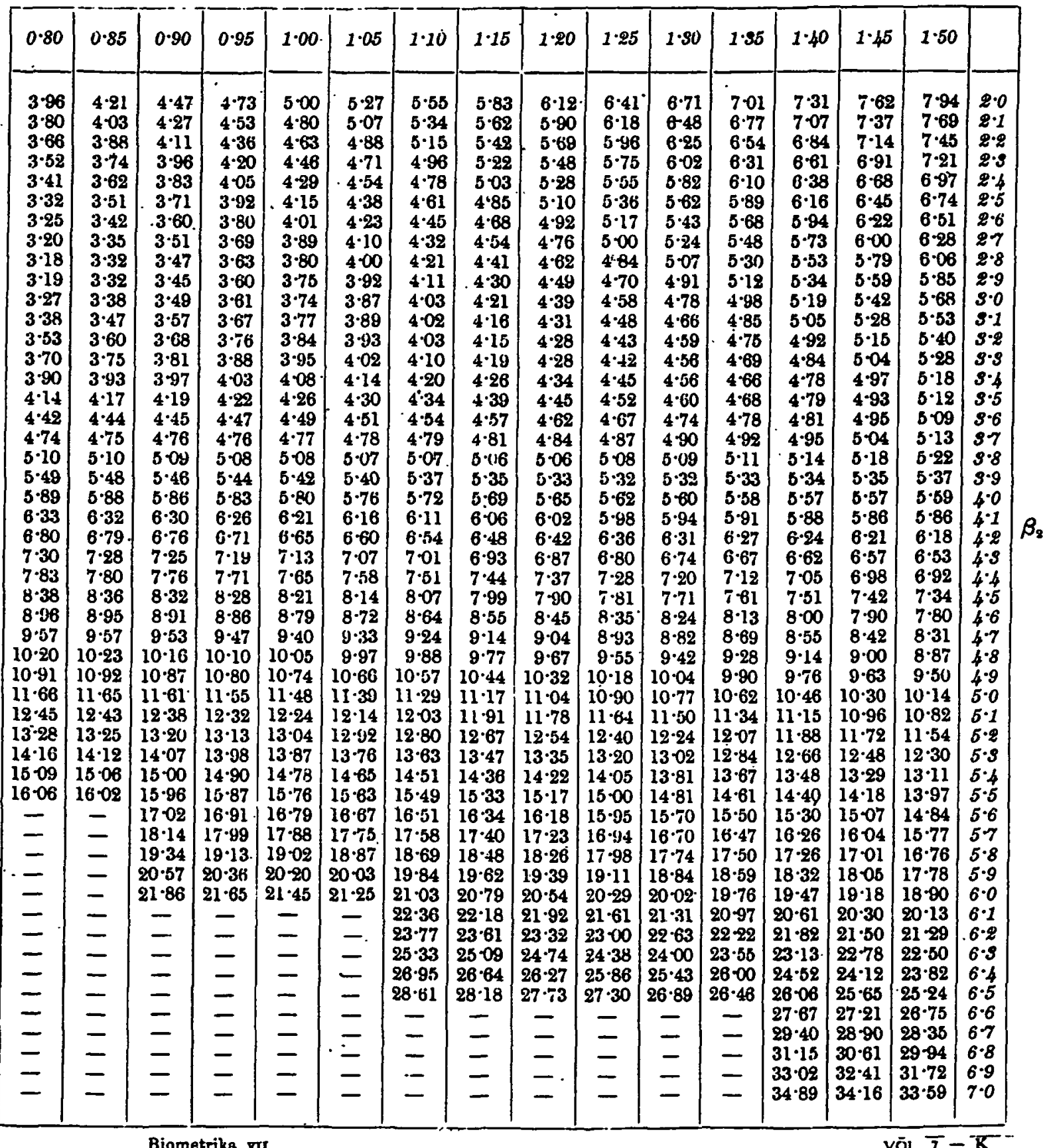


TABLE II:

Values of $\sqrt{N} \Sigma_{\beta_{2}}$.

$\beta_{1}$

\begin{tabular}{|c|c|c|c|c|c|c|c|c|c|c|c|c|c|c|c|c|}
\hline & 0.00 & 0.05 & $0 \cdot 10$ & 0.15 & 0.20 & 0.25 & 0.30 & 0.35 & 0.40 & .0 .45 & 0.50 & 0.55 & $0 \cdot \dot{60}$ & 0.65 & 0.70 & 0.75 \\
\hline $2 \cdot 0$ & 41 & 60 & 1.74 & 1.93 & $2 \cdot 11$ & 28 & $2 \cdot 44$ & $2 \cdot 60$ & $2 \cdot 77$ & 2:94 & $3 \cdot 12$ & $3 \cdot 29$ & $3 \cdot 46$ & 365 & 3.86 & \\
\hline & & 1.78 & & 200 & $2 \cdot 10$ & & $2 \cdot 35$ & $2 \cdot 51$ & $2 \cdot 68$ & 2.86 & 305 & $3 \cdot 24$ & 3.44 & 3.64 & 3.84 & 4.04 \\
\hline & 1.73 & 1.94 & & $2 \cdot 16$ & $8 \cdot 20$ & & $2 \cdot 40$ & $2 \cdot 53$ & $2 \cdot 68$ & 2.85 & $3: 04$ & $3 \cdot 23$ & 3.43 & & 3.83 & 403 \\
\hline & 1.95 & & & $\mathbf{2} \cdot \mathbf{3 5}$ & 2.42 & & 2.58 & 267 & $\mathbf{2} \cdot 78$ & 2.92 & 309 & $3 \cdot 27$ & 45 & 364 & 3.84 & 403 \\
\hline & $2 \cdot 1$ & & & $\overline{8} \cdot 60$ & $8 \cdot 78$ & 2.82 & 2.90 & $2-96$ & 302 & $3 \cdot 10$ & $3 \cdot 22$ & 3.35 & 3.50 & 3.68 & 3.86 & 4.03 \\
\hline & $2 \cdot 46$ & $8 \cdot 68$ & 8.83 & 8.97 & 3.00 & $3 \cdot 19$ & $3 \cdot 27$ & 3.31 & 3.32 & 3.36 & $3 \cdot 47$ & 3.53 & 3.63 & 3 & 3.88 & $4 n 3$ \\
\hline 2 & 278 & 30 & $3 \cdot 2$ & & 3.58 & & 3.66 & $3 \cdot 69$ & 369 & 3.70 & $3 \cdot 75$ & $3 \cdot 78$ & & 3. & & \\
\hline & $3 \cdot 17$ & $3 \cdot 48$ & $3 \cdot 7$ & 3.8 & & & & $4 \cdot 12$ & 4.1 & & 407 & & & & & 4. \\
\hline & 3.64 & & & 4.4 & & & & 4.5 & 4. & & 4.44 & & 4.3 & & $4 \cdot 31$ & 43 \\
\hline & 4.22 & $4 \cdot 6$ & 4. & 5.11 & 5.20 & & $5 \cdot 18$ & 5.13 & 307 & $4 \cdot 9$ & $4: 90$ & 4.80 & $4 \cdot 70$ & $4 \cdot 63$ & 4 & 46 \\
\hline & $4 \cdot 90$ & & & 5.8 & $5 \cdot 95$ & & 5.86 & 5.76 & & 5.53 & 8.42 & $5 \cdot 30$ & $5 \cdot 20$ & $5 \cdot 12$ & 5.05 & \\
\hline & $5 \cdot 75$ & & & & 6.90 & & & & & & 6.07 & 5.92 & 3.79 & & & 6.55 \\
\hline & & & & & 7.97 & & & $7 \cdot 49$ & $7 \cdot 21$ & 703 & 6.86 & $6 \cdot 70$ & 6.53 & 6.39 & & 6.14 \\
\hline & & & & & $0 \cdot 22$ & & & 8.53 & $8 \cdot 29$ & & $7 \cdot 83$ & $7 \cdot 60$ & $7 \cdot 38$ & $7 \cdot 20$ & 702 & \\
\hline & $9 \cdot 37$ & $10 \cdot 28$ & $10 \cdot 68$ & $10 \cdot 78$ & $10 \cdot 67$ & $10 \cdot 46$ & $10 \cdot 20$ & $9 \cdot 91$ & 962 & $9 \cdot 31$ & 0.01 & $8 \cdot 73$ & 8.44 & $8 \cdot 18$ & 7.92 & $7 \cdot 6$ \\
\hline & $10 \cdot 85$ & $11 \cdot 75$ & $12 \cdot 31$ & $12 \cdot 52$ & $2 \cdot 46$ & $18 \cdot 25$ & 11.95 & 1160 & $11 \cdot 24$ & 10.86 & $10 \cdot 45$ & 10.03 & 969 & 0.26 & & 8.54 \\
\hline$s$ & 67 & $13 \cdot 74$ & $14 \cdot 40$ & 14.78 & 14.53 & 14.21 & 13.80 & $13 \cdot 38$ & 95 & 12.55 & $18 \cdot 10$ & 11.60 & 11.06 & 10.54 & $10-02$ & 9.55 \\
\hline & $\cdot 78$ & 1598 & 16.78 & 1709 & 16.93 & $16 \cdot 53$ & 1605 & 15.38 & 1500 & 14.61 & 1408 & $13 \cdot 49$ & 18.74 & 202 & $11 \cdot 36$ & $10 \cdot 80$ \\
\hline 3 & .50 & 18.83 & 19.83 & 20.03 & $19 \cdot 78$ & $19 \cdot 36$ & 18.76 & $18 \cdot 22$ & $17 \cdot 64$ & 16.98 & $16 \cdot 25$ & $5 \cdot 30$ & $4 \cdot 48$ & 3.60 & $12 \cdot 88$ & $12 \cdot 27$ \\
\hline & $20 \cdot 80$ & $22 \cdot 50$ & $23 \cdot 68$ & $23 \cdot 81$ & 23 & 22.67 & 21.98 & $21 \cdot 14$ & & $19 \cdot 45$ & $18 \cdot 58$ & 17.54 & 6.50 & 5.54 & 14.77 & 1406 \\
\hline 40 & 24.74 & 26.83 & $28 \cdot 47$ & 28.05 & 27 & 3.29 & $25-25$ & 2418 & & & & & 4 & & & 16.36 \\
\hline $4 \cdot 1$ & - & - & 35.00 & $34 \cdot 17$ & $32 \cdot 88$ & $31 \cdot 36$ & $29 \cdot 77$ & $28 \cdot 13$ & $26 \cdot 60$ & $25 \cdot 12$ & & $22 \cdot 64$ & 21.54 & & $19 \cdot 56$ & 1862 \\
\hline $4: 2$ & - & - & $43 \cdot 3$ & $41 \cdot 4$ & $39 \cdot 2$ & 37.2 & $36 \cdot 2$ & $33-2$ & $31-2$ & $29-2$ & $27 \cdot 4$ & 26.0 & $24 \cdot 7$ & 23.4 & $22 \cdot 3$ & $21 \cdot 3$ \\
\hline $\begin{array}{l}4.8 \\
4.4\end{array}$ & $\bar{z}$ & $=$ & $\begin{array}{l}55 \cdot 3 \\
72 \cdot 7\end{array}$ & $\begin{array}{l}51 \cdot 6 \\
88 \cdot 0\end{array}$ & $\begin{array}{l}48 \cdot 0 . \\
59.7\end{array}$ & $\begin{array}{l}44 \cdot 6 \\
54 \cdot 1\end{array}$ & $\begin{array}{l}41 \cdot 2 \\
49 \cdot 5\end{array}$ & $\begin{array}{l}38 \cdot 6 \\
45 \cdot 7\end{array}$ & $\begin{array}{c}36 \cdot 2 \\
42 \cdot 1\end{array}$ & $\begin{array}{l}33.8 \\
39-2\end{array}$ & $\begin{array}{l}31 \cdot 8 \\
36 \cdot 8\end{array}$ & $\begin{array}{l}30 \cdot 1 \\
34 \cdot 8\end{array}$ & 28.5 & 26.9 & $\begin{array}{l}25 \cdot 6 \\
29 \cdot 2\end{array}$ & $\begin{array}{l}24.3 \\
97.7\end{array}$ \\
\hline 4.5 & - & $=$ & 96.5 & $82 \cdot 7$ & 72.7 & 65.3 & 59.8 & 54.7 & & $47-2$ & 440 & & 38.5 & & $34 \cdot 1$ & 320 \\
\hline & - & - & - & - & - & - & 750 & 680 & & 56.9 & $52 \cdot 2$ & 48.2 & $45 \cdot 1$ & 42 & 396 & $37-2$ \\
\hline & - & - & - & - & $\because$ & - & $101 \cdot 3$ & & 76.8 & $68 \cdot 3$ & 620 & $56 \cdot 9$ & 527 & $49 \cdot 1$ & $45 \cdot 9$ & $42 \cdot 8$ \\
\hline & I & - & - & - & - & - & & 1158 & $\begin{array}{r}96 \cdot 2 \\
\end{array}$ & 826 & 727 & & $60-9$ & & 52.9 & $49 \cdot 3$ \\
\hline 4 & - & - & - & - & - & - & 204.5 & 150.8 & $122-3$ & $102 \cdot 5$ & $89 \cdot 1$ & & & & $61 \cdot 4$ & 567 \\
\hline 5 & - & - & - & - & - & - & $325 \cdot 7$ & 206.0 & $154 \cdot 2$ & 126.8 & 110.1 & 96.9 & & & $71 \cdot 2$ & $65 \cdot 6$ \\
\hline & - & - & - & - & - & - & - & - & - & - & - & - & & & & 780 \\
\hline & - & - & - & - & - & - & - & - & - & - & - & - & & & & 910 \\
\hline & - & - & - & - & - & - & - & - & & - & - & - & & & & $109 \cdot 6$ \\
\hline 5 & - & - & - & - & - & - & - & - & & - & - & - & & & & $132 \cdot 6$ \\
\hline & - & & - & - & - & - & - & - & & - & - & - & $340: 8$ & 2460 & $195 \cdot 3$ & $163 \cdot 2$ \\
\hline 5 & - & - & - & - & - & - & - & - & & & - & - & $\div$ & - & - & - \\
\hline & & & - & & - & & & & & & & & & & & \\
\hline & - & & & - & & & - & & & - & & - & & & - & \\
\hline & & & & - & & & & & & & & & & & - & \\
\hline & & & - & - & & & 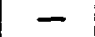 & & & & & - & & & - & - \\
\hline & & & & & & & & & & & & & & & & \\
\hline & & & & & & & 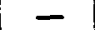 & & & & & & & & & \\
\hline & & & & & & & & & & & & & & & & \\
\hline & & & & & & & & & & & & & & & & \\
\hline & & & & & & & & & & & & & & & & \\
\hline & & & & & & & & & & & & & & & & \\
\hline & & & & & & & & & & & & & & & & \\
\hline & & & 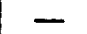 & & & & & & & & & & & & & - \\
\hline & - & $=$ & - & & - & & & - & & - & - & - & - & - & - & - \\
\hline & - & - & - & - & - & - & & - & - & - & & - & - & - & - & - \\
\hline
\end{tabular}


TABLE II.-(continued).

Values of $\sqrt{N} \Sigma_{\beta_{1}}$.

$\beta_{1}$

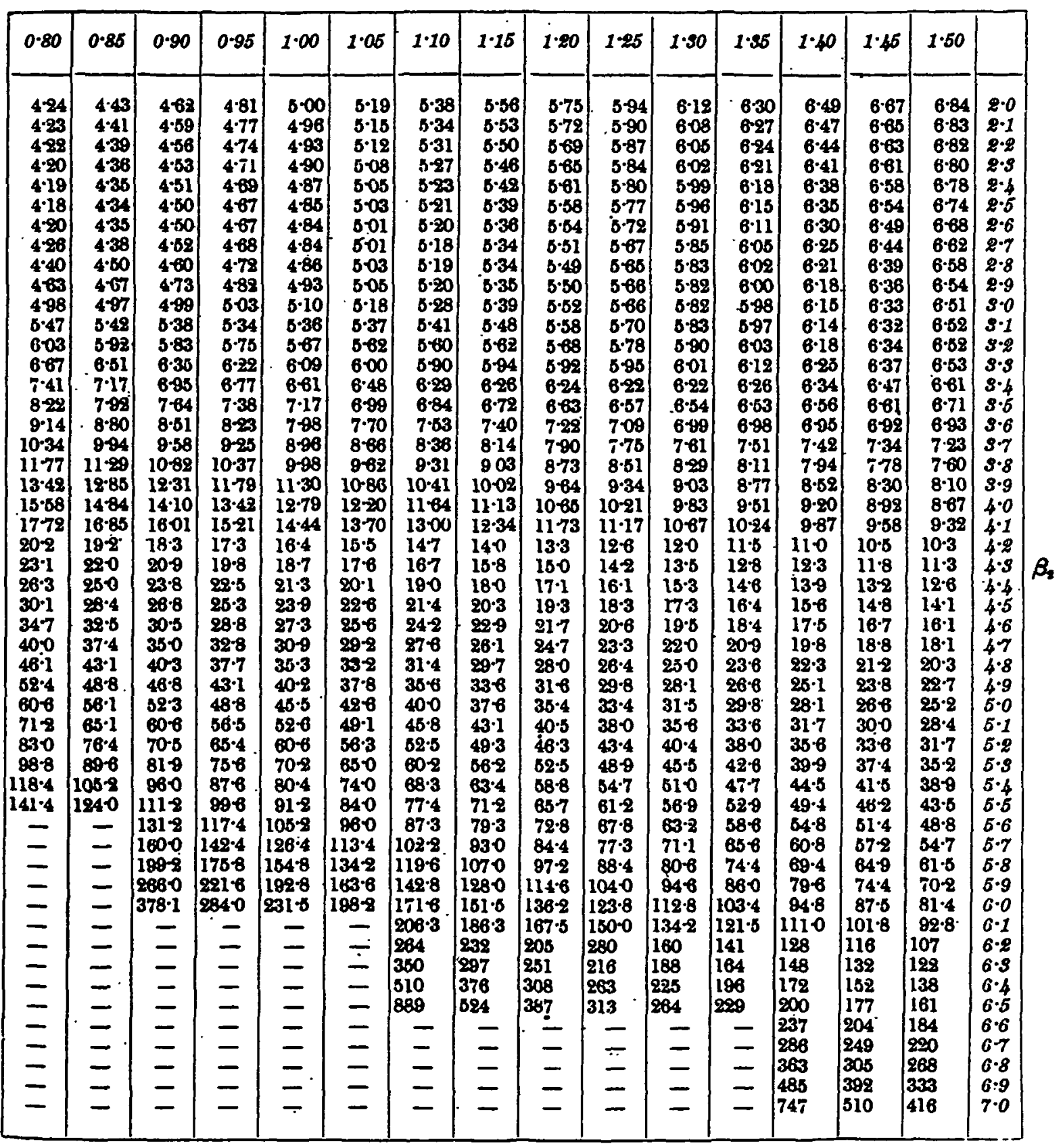


TABLE III:

Valiues of . $R_{\beta_{3} \beta_{1}}$.

$\beta_{1}$

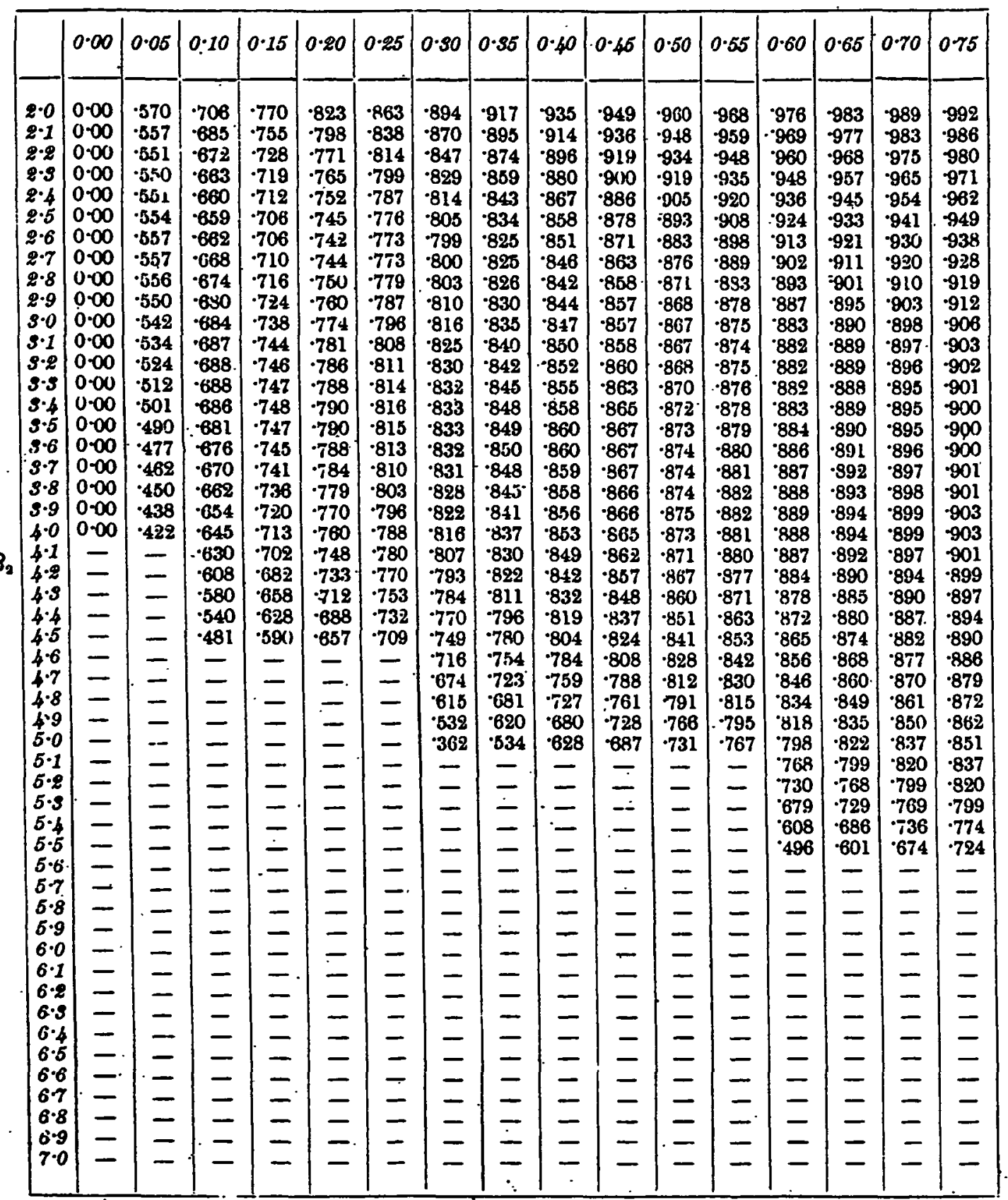


TABLE III.-(continued)

Values of $R_{p_{1} p_{2}}$.

$\boldsymbol{\beta}_{\mathbf{1}}$

\begin{tabular}{|c|c|c|c|c|c|c|c|c|c|c|c|c|c|c|c|}
\hline 0.80 & 0.85 & 0.90 & 0.95 & 1.00 & 1.05 & $1 \cdot 10$ & $1 \cdot 15$ & 1.20 & 1.25 & 1.50 & 1.55 & 1.40 & 1.45 & 1.50 & \\
\hline-993 & -995 & .897 & 999 & 1000 & 1.000 & 1.000 & 1000 & 1000 & 1.000 & 1.000 & $1-000$ & $1 \cdot 000$ & 1.000 & 1.000 & $2 \cdot 0$ \\
\hline-989 & 901 & 994 & -996 & 908 & $\cdot 998$ & $\cdot 999$ & -990 & .999 & $1 \cdot 100$ & 1.000 & $1 \cdot 000$ & 1.000 & 1.000 & 1.000 & $\tilde{z} \cdot \mathbf{1}$ \\
\hline .983 & 986 & 989 & .992 & -995 & 996 & 997 & 998 & .998 & -999 & 1.000 & 1.000 & 1.000 & 1.000 & 1.000 & $2 \cdot 2$ \\
\hline-976 & 980 & .984 & .988 & $\cdot 992$ & 993 & 994 & 995 & 997 & 998 & 999 & -999 & 999 & 1000 & $-1 \cdot 000$ & 2.3 \\
\hline 968 & -973 & 978 & 983 & -987 & 989 & 991 & -993 & 995 & 996 & -998 & -998 & 999 & $\begin{array}{r}999 \\
.009\end{array}$ & $1 \cdot 000$ & $\begin{array}{c}8.4 \\
0.5\end{array}$ \\
\hline .958 & 965 & 972 & .977 & .982 & -985 & 988 & 990 & 992 & -994 & 996 & -997 & 998 & 999 & $1 \cdot 000$ & $\begin{array}{l}2.5 \\
0.6\end{array}$ \\
\hline .947 & 956 & .964 & .970 & .976 & .980 & 984 & .986 & .988 & 991 & .993 & .995 & 997 & "998 & 999 & 26 \\
\hline 937 & 947 & 957 & .963 & 968 & $\begin{array}{r}973 \\
-965\end{array}$ & 977 & $\begin{array}{l}980 \\
.974\end{array}$ & $\begin{array}{r}-983 \\
.978\end{array}$ & $\begin{array}{l}986 \\
.981\end{array}$ & $\begin{array}{l}-989 \\
-095\end{array}$ & -997 & $\begin{array}{l}995 \\
-0999\end{array}$ & $\begin{array}{r}999 \\
.994\end{array}$ & $\begin{array}{r}998 \\
.996\end{array}$ & $\begin{array}{l}2.7 \\
2 \cdot 8\end{array}$ \\
\hline $\begin{array}{r}9988 \\
-921\end{array}$ & $\begin{array}{r}-939 \\
-932\end{array}$ & $\begin{array}{r}949 \\
-942\end{array}$ & $\begin{array}{l}955 \\
-947\end{array}$ & $\begin{array}{r}960 \\
-952\end{array}$ & $\begin{array}{r}965 \\
.957\end{array}$ & 970 & $\begin{array}{r}974 \\
-963\end{array}$ & $\begin{array}{r}978 \\
972\end{array}$ & $\begin{array}{l}981 \\
-970\end{array}$ & $\begin{array}{l}-985 \\
-980\end{array}$ & $\begin{array}{r}-989 \\
-984\end{array}$ & $\begin{array}{r}992 \\
-988\end{array}$ & $\begin{array}{l}994 \\
990\end{array}$ & $\begin{array}{r}996 \\
.992\end{array}$ & 2.9 \\
\hline .915 & .923 & 931 & -937 & -943 & 948 & -954 & .960 & 968 & -971 & .975 & -979 & 993 & -986 & .988 & $s .0$ \\
\hline 909 & 915 & $9 z z$ & 929 & $\cdot 936$ & 942 & -947 & -953 & .959 & $\cdot 965$ & $\cdot 970$ & $\cdot 974$ & 978 & $\cdot 981$ & 984 & $s \cdot 1$ \\
\hline 907 & 912 & 918 & .924 & 933. & $\cdot 936$ & -941 & ·946 & .952 & $\cdot 958$ & .963 & 968 & 973 & -977 & 980 & $3 \cdot 2$ \\
\hline .906 & :909 & :914 & 919 & $y 25$ & -930 & •935 & $-9 \pm 0$ & •946 & 951 & .936 & -961 & 966 & .971 & 975 & $3 \cdot s$ \\
\hline 905 & .808 & 912 & 916 & 920 & 925 & 930 & 033 & .940 & $-9 \pm 5$ & .950 & .934 & .958 & -964 & 974 & 5.4 \\
\hline .904 & 907 & 910 & .914 & 918 & 922 & 926 & .931 & $\cdot 936$ & $\cdot 940$ & $\cdot 944$ & .948 & •952 & .958 & 965 & 3.5 \\
\hline & 907 & 910 & .014 & 918 & .921 & -924 & .028 & .932 & .935 & .038 & .942 & 946 & -052 & 959 & 3.6 \\
\hline 905 & 807 & 910 & .914 & 917 & .920 & 923 & .927 & $\cdot 930$ & .933 & .935 & 937 & .940 & .946 & 953 & 37 \\
\hline 905 & 908 & .911 & 914 & 7 & $\cdot 920$ & •922 & .925 & .928 & -930 & $\cdot 932$ & 934 & .936 & .941 & 948 & 3.8 \\
\hline 906 & 909 & 911 & .914 & .917 & 919 & 921 & 924 & .927 & .929 & .931 & -933 & 935 & .939 & 944 & 3.9 \\
\hline .908 & .909 & 912 & -914 & .917 & 919 & 921 & .923 & 96 & .928 & -930 & -932 & •934 & 936 & 940 & 40 \\
\hline 905 & 918 & 911 & 914 & 917 & - 9 ro & 921 & .923 & .925 & 927 & -0.30 & 931 & 932 & $\cdot 933$ & 934 & 4.1 \\
\hline 905 & דi & 910 & 913 & 916 & 919 & $\begin{array}{l}-921 \\
.990\end{array}$ & 923 & .924 & 926 & .929 & $\begin{array}{r}-929 \\
-028\end{array}$ & 930 & $\begin{array}{l}930 \\
.0907\end{array}$ & 929 & $4 \%$ \\
\hline 903 & 906 & 910 & 913 & 916 & 918 & 920 & 922 & 924 & 926 & .929 & 928 & 928 & 927 & 924 & 4.8 \\
\hline 9900 & $\cdot 904$ & 908 & .912 & 916 & 918 & 920 & 922 & 923 & 926 & .928 & .927 & 927 & 925 & 922 & $4: 4$ \\
\hline 8997 & 902 & 906 & 910 & 915 & $\cdot 918$ & 920 & 922 & .923 & y26 & .928 & 927 & $\cdot 926$ & 923 & 920 & 4.5 \\
\hline .893 & -898 & 003 & .908 & $\cdot 913$ & -916 & 919 & $\cdot 920$ & 022 & 925 & .327 & .926 & 925 & 923 & 920 & 40 \\
\hline & .894 & .800 & .905 & .910 & .913 & 917 & .919 & .921 & 924 & .920 & .925 & 925 & .923 & 922 & 47 \\
\hline$\cdot 8$ & 8910 & 896 & .901 & .906 & 910 & 914 & 917 & .920 & .923 & 925 & .926 & .926 & .923 & 925 & 4.8 \\
\hline & .884 & $\cdot 890$ & 895 & 901 & 907 & •911 & .915 & .919 & .922 & .925 & -926 & .927 & .927 & •928 & 499 \\
\hline & $\cdot 875$ & ${ }^{8} 883$ & $\cdot 889$ & 896 & .903 & 908 & .913 & .918 & .922 & .923 & & .928 & •930 & '93z & $5 \cdot 0$ \\
\hline & -864 & 875 & -882 & $\cdot 800$ & 898 & 905 & .911 & .917 & -922 & .92 .5 & -928 & $\cdot 931$ & .933 & 936 & $5 \cdot 1$ \\
\hline .837 & 852 & ${ }^{\circ} 866$ & -875 & $\cdot 884$ & $\cdot 892$ & .901 & .909 & .916 & 921 & .924 & .928 & .933 & '937 & 941 & $5 \cdot 2$ \\
\hline 820 & 8390 & 853 & 865 & 876 & 885 & 895 & .904 & .913 & 918 & $\cdot 023$ & .929 & 935 & 9940 & 945 & 5.8 \\
\hline 798 & 818 & 8.37 & 853 & 867 & 877 & '888 & $\cdot 898$ & $\cdot 9 x$ & .91 & .921 & -928 & 935 & $\cdot 9$ & 947 & 5.4 \\
\hline$\cdot 764$ & 702 & 817 & .837 & 854 & & 880 & 890 & .900 & .910 & 918 & -925 & •933 & 940 & .947 & $5 \cdot 5$ \\
\hline & - & 789 & $\cdot 815$ & & & 888 & $\cdot 880$ & .890 & .904 & .911 & -917 & 926 & -935 & 944 & $5 \cdot 6$ \\
\hline & & $7: 0$ & 786 & 811 & & -854 & -869 & $\cdot 880$ & 8992 & .901 & -909 & 917 & 927 & .938 & $5 \cdot 7$ \\
\hline & - & 7701 & 748 & •783 & 8811 & •835 & • 852 & 866 & 879 & • 890 & $\cdot 897$ & 905 & $\cdot 915$ & •928 & 5.8 \\
\hline & - & $\cdot 640$ & $\cdot 700$ & $\cdot 748$ & . 781 & •810 & .828 & .846 & $• 861$ & .875 & -883 & 892 & 90 & •913 & $\cdot 9$ \\
\hline & - & ·544 & -639 & ${ }^{\cdot} 70.3$ & 746 & 778 & 802 & .825 & $\cdot 842$ & $\cdot 857$ & .867 & 879 & $\cdot 886$ & .893 & 6.0 \\
\hline- & - & - & - & - & - & ${ }^{7} 741$ & $\cdot 769$ & 796 & $\cdot 820$ & .837 & $\cdot 852$ & -868 & $\cdot 872$ & •873 & $6 \cdot 1$ \\
\hline & & & & & 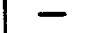 & •691 & 727 & $\cdot 762$ & 792 & -815 & -836 & '852 & 858 & 856 & 6.2 \\
\hline & - & - & - & & & 628 & 678 & $\cdot 724$ & 761 & 790 & -818 & & $\cdot 845$ & 842 & 6.8 \\
\hline & & - & & & & 32 & 6008 & 67 & 724 & 763 & 793 & 818 & 831 & ${ }^{\circ} 834$ & 0.4 \\
\hline & - & - & - & & & 35 & 326 & 618 & 680 & 726 & 761 & ${ }^{7} 791$ & •814 & •831 & .5 \\
\hline & &. & - & & & & & & & & & $\cdot 761$ & 780 & '832 & $\cdot 6$ \\
\hline & & & & & & & & & & - & & 721 & $\cdot 760$ & -837 & $\cdot 7$ \\
\hline & - & - & - & - & & - & - & - & - & - & - & 670 & $\cdot 727$ & 845 & 6.8 \\
\hline - & - & -- & - & - & - & - & - & - & - & - & - & .600 & -683 & $\cdot 857$ & 6.9 \\
\hline- & & & & - & - & - & - & - & - & - & - & $\cdot 468$ & 602 & 876 & $7 \cdot 0$ \\
\hline
\end{tabular}


to

\begin{tabular}{|c|c|}
\hline 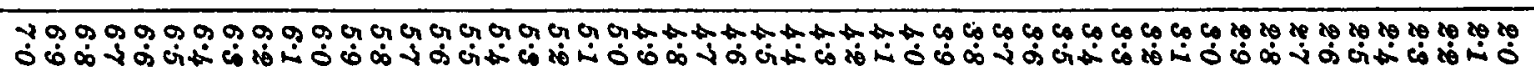 & \\
\hline 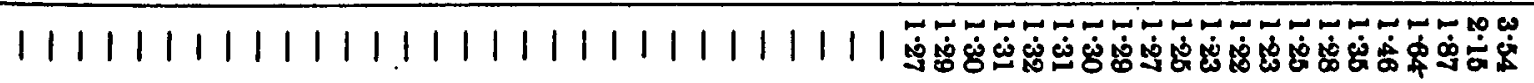 & $\dot{8}$ \\
\hline 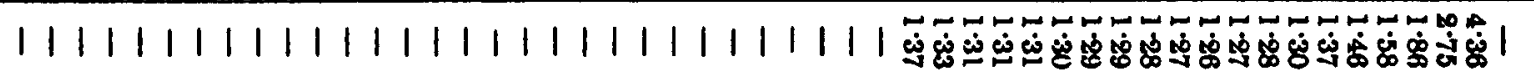 & \&̊․ \\
\hline 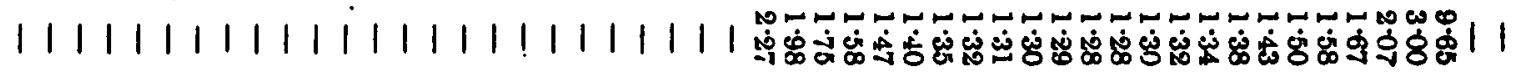 & $\stackrel{1}{\circ}$ \\
\hline 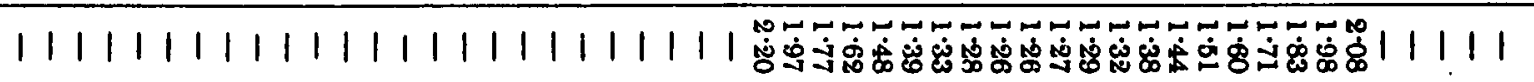 & $\stackrel{\leftrightarrow}{\leftrightarrow}$ \\
\hline 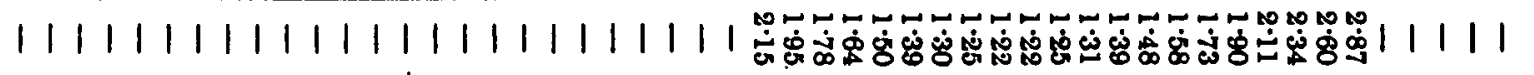 & i̊ \\
\hline 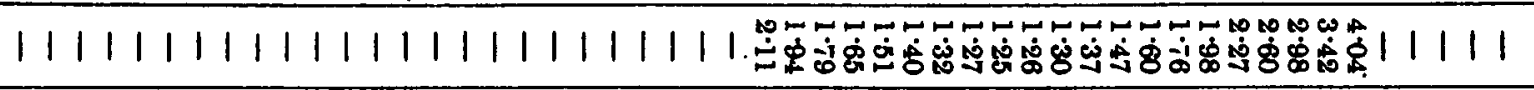 & 里 \\
\hline 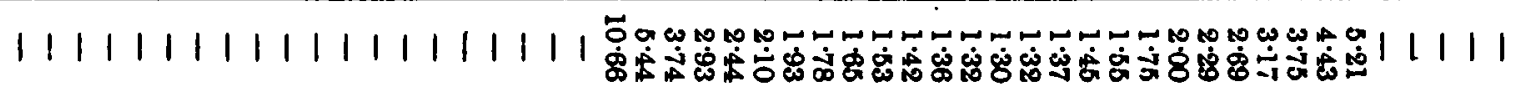 & $\dot{8}$ \\
\hline 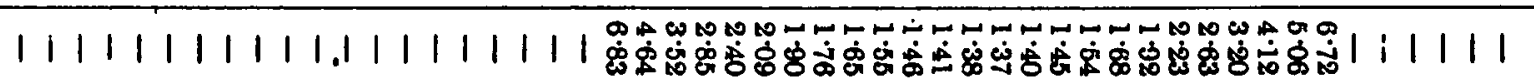 & \&ُو \\
\hline 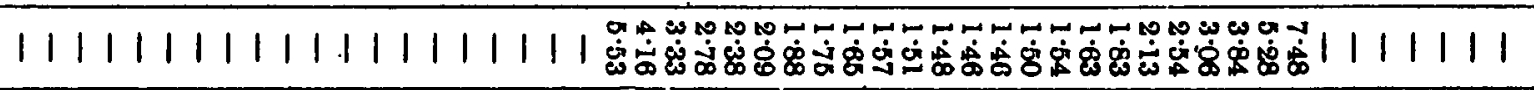 & ثें \\
\hline 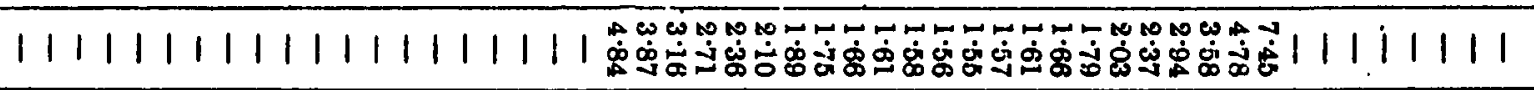 & हैं \\
\hline 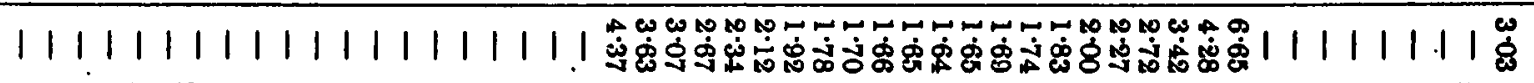 & :̊․ \\
\hline $111|1| 1|1| 1|1| 1|1| 1 \mid \begin{array}{l}0 \\
0\end{array}$ & \& \\
\hline 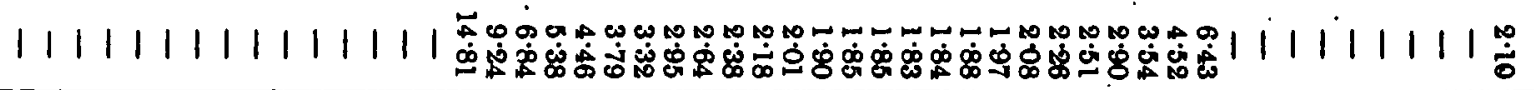 & $\stackrel{\circ}{\circ}$ \\
\hline 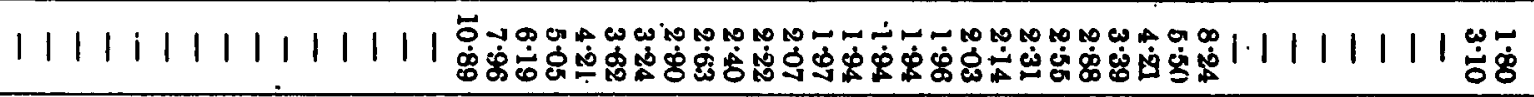 & \& \\
\hline 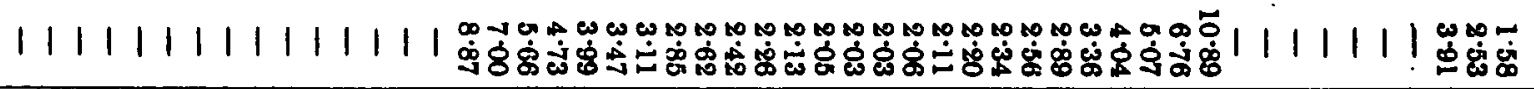 & à \\
\hline 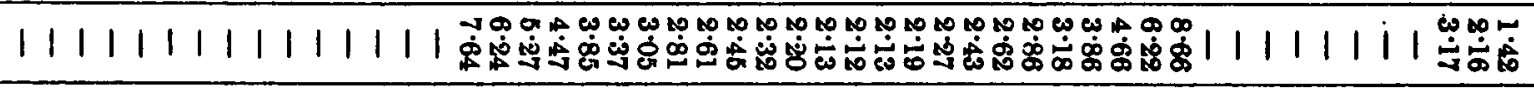 & $\begin{array}{l}0 \\
0 \\
0\end{array}$ \\
\hline 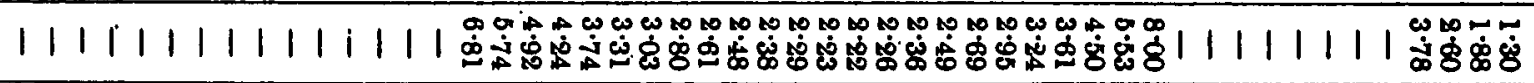 & क्ष \\
\hline
\end{tabular}


TABLE IV.-(continuea).

Values of $\frac{\sqrt{N}}{\sigma} \Sigma_{\mathrm{d}}$.

$\beta_{1}$

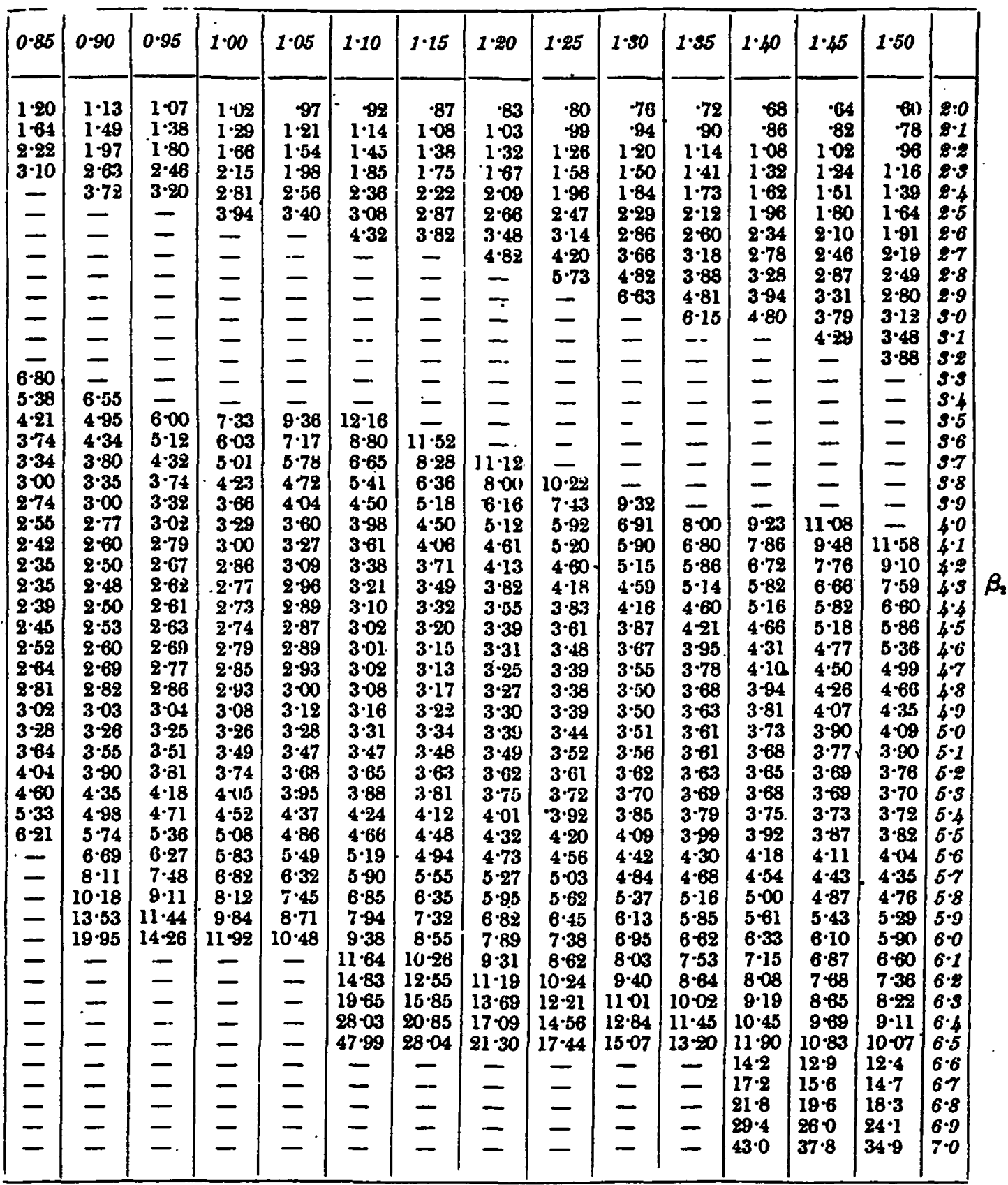


TABLE $\nabla$.

Values of $\sqrt{N} \Sigma_{\text {ut }}$.

\begin{tabular}{|c|c|c|c|c|c|c|c|c|c|c|c|c|c|c|c|c|}
\hline \multicolumn{17}{|c|}{$\beta_{1}$} \\
\hline & 0.00 & 0.05 & $0 \cdot 10$ & 0.15 & $0.20 \mid$ & 0.25 & 0.30 & 0.25 & 0.40 & 0.45 & 0.50 & 0.55 & 0.60 & 0.65 & 0.70 & 075 \\
\hline & & & & - & $\pi$ & $\overline{-}$ & $\pi$ & - & 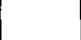 & - & $3 \cdot 41$ & 9.80 & 9.43 & & $\cdot 89$ & \\
\hline & & $4 \cdot 20$ & & - & $=$ & $=$ & $\bar{Z}$ & $\bar{z}$ & 二 & Z & - & - & - & 3.67 & 3.02 & 2.58 \\
\hline & & $2 \cdot 63$ & $9 \cdot 50$ & - & - & - & - & - & - & - & - & - & - & - & 5.57 & $4 \cdot 10^{\circ}$ \\
\hline & 1.64 & $1 \cdot 78$ & & - & - & - & - & - & - & - & - & - & - & - & -1 & - \\
\hline & 1.46 & 1.49 & 2.02 & - & 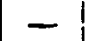 & - & - & - & - & - & - & - & - & - & - & \\
\hline & 1.35 & 1.41 & 1.62 & 2.02 & $2 \cdot 80$ & 4.06 & 5.08 & - & - & - & - & - & - & - & - & - \\
\hline & $\mathrm{I} \cdot 28$ & 130 & 1.43 & $1 \cdot 75$ & & $2 \cdot 82$ & 3.65 & $4 \cdot 96$ & - & & & - & - & - & 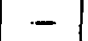 & \\
\hline 2 & $1-25$ & $1 \cdot 25$ & & 1 . & $\mathrm{I} \cdot 84$ & $2 \cdot 29$ & $2 \cdot 85$ & & 4.84 & & & - & . & - & - & - \\
\hline & & $1-z z$ & & & 1.59 & $1 \cdot 89$ & & $2 \cdot 70$ & & & & & & - & & \\
\hline & & & & & $1 \cdot 43$ & 1.63 & 1.86 & $2 \cdot 19$ & $2 \cdot 6$ & & $4 \cdot 30$ & & & & & \\
\hline & & & & & $1 \cdot 34$ & & & 1.84 & $2 \cdot 12$ & & 300 & 3.76 & $4 \cdot 62$ & 6.02 & $\cdot 12$ & \\
\hline & & $1 \cdot 2 z$ & & & $1 \cdot 27$ & $1 \cdot 36$ & 1.50 & $1 \cdot 67$ & 1.88 & & 2.50 & & & $4 \cdot 72$ & -10 & 8.08 \\
\hline & $1 \cdot 27$ & $1-23$ & $1 \cdot 22$ & & $1 \cdot 23$ & $1 \cdot 29$ & 1. & & $1 \cdot 70$ & & & & & & $4 \cdot 87$ & .42 \\
\hline & & $1 \cdot 25$ & $1 \cdot 23$ & & 1.21 & 1.24 & $1 \cdot 33$ & 44 & .1 .58 & & & & & & 88 & 1.53 \\
\hline & $1 \cdot 3$ & $1 \cdot 27$ & $\mathrm{l} \cdot 24$ & 1.21 & $1 \cdot 20$ & $1 \cdot 23$ & 1.29 & $1 \cdot 38$ & 49 & & & 4 & & $2 \cdot 75$ & 18 & \\
\hline & & 1.20 & $1 \cdot 25$ & $1 \cdot 21$ & 1.20 & 1.22 & 1.28 & 1.35 & $1 \cdot 43$ & & & 1.87 & 2.09 & $2 \cdot 37$ & 68 & \\
\hline 3 & $1 \%$ & $1 \cdot 30$ & $1 \cdot 26$ & $1 \cdot 22$ & 1.20 & $1 \cdot 22$ & 1.26 & 1.32 & 1.40 & .50 & & 1.75 & 1.91 & $2 \cdot 13$ & 39 & 65 \\
\hline 3 & $1 \cdot 31$ & $1 \cdot 31$ & $1 \cdot 28$ & $1 \cdot 24$ & 1.20 & 1.23 & 1.27 & 1.32 & 1.39 & 47 & & 1.67 & 1.80 & .1 .08 & $2 \cdot 19$ & $2 \cdot 40$ \\
\hline 3. & $1 \cdot 30$ & $1 \cdot 3 z$ & 1.30 & $1 \cdot 27$ & 1.23 & 1.25 & 1.28 & 1.33 & 1.38 & & & 1.6 & & 1.88 & & $2 \cdot 22$ \\
\hline$s$ & $1 \cdot 29$ & $1 \cdot 34$ & 1.33 & $1 \cdot 30$ & $\mathrm{i} \cdot 28$ & 1.28 & & & & & & & & & & $2 \cdot 12$ \\
\hline 4.0 & $1-27$ & 1.36 & & 1. & $i \cdot 35$ & 1.35 & & & & & & & & & & 04 \\
\hline $4 \cdot 1$ & - & - & & & 45 & & & & & & & & & & & 03 \\
\hline $4: 2$ & - & - & & 1.57 & 1.57 & & 1. & & & & & & & & & 02 \\
\hline 4.3 & - & - & & 174 & 1.73 & 1. & $i$. & & & & & & & & & 2.06 \\
\hline & - & - & $1 \cdot 95$ & & 1.92 & & & & & & & & & 2.01 & & $2 \cdot 12$ \\
\hline & - & - & $2 \cdot 28$ & $2 \cdot 19$ & $2 \cdot 13$ & 2.09 & & & & & & & & & & .23 \\
\hline & - & - & - & - & - & - & 2.48 & & & & & & & 3. 32 & & \\
\hline & - & - & - & - & - & - & & 2.03 & $2 \cdot 8$ & & & $2 \cdot 6$ & & $2 \cdot 5$ & & \\
\hline 4 & - & - & - & - & - & - & 3.78 & 3.53 & $3 \cdot 3$ & 3.21 & & $2 \cdot 97$ & & $2 \cdot 8$ & & \\
\hline $4^{\circ}$ & - & - & - & - & - & - & 5.48 & & & $3 \cdot 87$ & & 3.44 & & 3. & 7 & 3.01 \\
\hline 5 & - & - & - & - & - & - & $11 \cdot 12$ & 6.96 & 3.52 & $4 \cdot 82$ & $4 \cdot 36$ & 4.02 & & & & \\
\hline 5 & - & - & - & - & - & - & - & - & - & - & 100 & - & & & 400 & \\
\hline 5 & - & - & - & - & - & - & - & - & - & - & - & - & & & & \\
\hline 5 & & - & - & - & - & - & - & - & - & - & - & - & & 6.18 & & \\
\hline 5 & - & - & - & - & - & - & - & - & - & - & - & - & $\theta$ & $7 \cdot 76$ & 6.80 & \\
\hline & - & - & - & - & - & - & - & - & - & - & - & - & 14.80 & $10 \cdot 67$ & 8.87 & 7.71 \\
\hline & & - & & - & - & & - & - & - & & - & - & - & - & -1 & - \\
\hline & & & & & & & - & - & & & & - & & & & \\
\hline & - & & & & & & - & - & & & & - & & & & \\
\hline & - & & & & & & & & & & & & & & & \\
\hline & - & & - & & & & & & & & - & - & & & & \\
\hline & - & & & & & & - & - & - & - & - & - & - & & - & - \\
\hline & - & & - & - & - & - & - & - & - & - & - & - & - & - & - & - \\
\hline 6 & - & - & - & & - & - & - & - & - & - & - & - & - & - & - & - \\
\hline & - & - & - & & - & - & - & - & - & - & - & & & & - & - \\
\hline 6 & - & - & & & - & - & - & - & - & - & - & - & - & & - & - \\
\hline & - & & & - & - & - & - & - & - & - & - & & - & & - & - \\
\hline & $=$ & & & &. & - & & - & - & - & - & - & & & - & - \\
\hline & - & - & & & - & - & & - & - & - & - & - & - & - & - & - \\
\hline & - & & & & & - & & - & 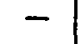 & & - & - & - & & - & - \\
\hline & - & & & - & - & - & - & - & - & - & - & & - & - & - & - \\
\hline
\end{tabular}


TABLE V.-(continued).

Values of $\sqrt{N} \Sigma_{\text {al }}$.

$\boldsymbol{\beta}_{\mathbf{1}}$

\begin{tabular}{|c|c|c|c|c|c|c|c|c|c|c|c|c|c|c|c|}
\hline 0.80 & 0.85 & 0.90 & 0.95 & 1.00 & 1.05 & $1 \cdot 10$ & $1 \cdot 15$ & $1 \cdot 20$ & $1 \cdot 25$ & $1 \cdot 30$ & 1.35 & 1.40. & $1 \cdot 45$ & 1.50 & \\
\hline $1 \cdot 59$ & 1.48 & $1 \cdot \mathbf{3 9}$ & & 124 & $1 \cdot 19$ & $1 \cdot 14$ & $1 \cdot 10$ & 106 & 102 & 99 & .95 & 91 & 87 & & 200 \\
\hline & & 1.80 & & 1.58 & & & & & 1.32 & & 1.20 & 1.15 & 1.10 & 105 & $z-1$ \\
\hline $3 \cdot 22$ & 2.65 & -2-29 & 2.08 & 198 & 1.91 & 1.84 & 1.78 & $1 \cdot 72$ & 1.66 & 1.59 & 1.52 & $1 \cdot 45$ & $1 \cdot 38$ & 1.31 & $2 \cdot 2$ \\
\hline $3 \cdot 23$ & 3.80 & 3.04 & $2 \cdot 75$ & 2.33 & $2 \cdot 40$ & $2 \cdot 30$ & $2 \cdot 21$ & $2 \cdot 12$ & 2.03 & 1.94 & 1.85 & 1.76 & 167 & 1.58 & $2 \cdot 3$ \\
\hline- & - & $4 \cdot 29$ & 3.64 & 3.31 & $3 \cdot 12$ & 2.94 & $2 \cdot 78$ & 263 & $2 \cdot 49$ & $2 \cdot 36$ & $2 \cdot 23$ & $2 \cdot 10$ & 1.28 & 1.86 & $2 \cdot 4$ \\
\hline- & - & - & - & $4 \cdot 77$ & $4 \cdot 27$ & 3.84 & 3.55 & $3 \cdot 29$ & 3.06 & 2.85 & $2 \cdot 66$ & $2 \cdot 49$ & $2 \cdot 32$ & $2 \cdot 15$ & 2.5 \\
\hline- & - & - & - & - & - & 5.72 & $5 \cdot 00$ & $4 \cdot 39$ & $3 \cdot 94$ & .3 .54 & 3.20 & $2 \cdot 93$ & 2.67 & $2 \cdot 44$ & 26 \\
\hline- & - & - & - & - & - & - & - & 6.25 & 5.20 & $4 \cdot 46$ & 3.88 & 3.42 & 308 & $2 \cdot 74$ & 27 \\
\hline- & - & - & - & - & & & - & - & $7 \cdot 0.5$ & 668 & $4 \cdot 78$ & 4.03 & 3.54 & 305 & $2 \cdot 8$ \\
\hline & & - & - & - & - & - & - & - & - & $7 \cdot 45$ & $6 \cdot 00$ & 4.85 & $4 \cdot 11$ & $\begin{array}{l}3 \cdot 37 \\
0.77\end{array}$ & $2 \cdot 9$ \\
\hline & - & - & - & - & - & - & - & - & - & - & $7 \cdot 80$ & 6.05 & $4 \cdot 77$ & 370 & 90 \\
\hline & $\bar{z}$ & - & $=$ & - & - & $\bar{z}$ & - & - & - & - & - & - & b.57 & $4 \cdot 10$ & $3 \cdot 1$ \\
\hline${ }_{30}$ & $6 \cdot \overline{624}$ & $\bar{z}$ & $=$ & $\bar{z}$ & $\bar{I}$ & $=$ & Z & $\bar{z}$ & $z$ & Z & Z & $\bar{z}$ & $\bar{z}$ & 4.58 & 3.8 \\
\hline 16 & 4.71 & $5 \cdot 60$ & - & $=$ & $\overline{-}$ & - & $\overline{-}$ & - & - & $\bar{z}$ & $\bar{z}$ & $\bar{z}$ & $=$ & 二 & 8.4 \\
\hline & $\begin{array}{l}3.89 \\
3 \\
3.29\end{array}$ & $4 \cdot 39$ & 5:58 & 6.85 & $8 \cdot 38$ & $11 \cdot 48$ & $\overline{0}$ & - & - & - & - & - & - & - & $s \cdot \bar{b}$ \\
\hline $\begin{array}{l}2 \cdot 95 \\
0 \cdot 64\end{array}$ & 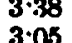 & 3.99 & $\begin{array}{l}4.66 \\
3.967\end{array}$ & $\begin{array}{r}5.50 \\
4.58\end{array}$ & $\begin{array}{l}6.48 \\
6.98\end{array}$ & $7 \cdot 53$ & $\begin{array}{l}9 \cdot 92 \\
7 \cdot 3\end{array}$ & $\overline{0.48}$ & $\bar{z}$ & $\bar{z}$ & $\bar{z}$ & - & $=$ & 二 & 36 \\
\hline 67 & 3.05 & $3 \cdot 49$ & 3.97 & 4.58 & 6.22 & 6.00 & $7 \cdot 36$ & & - & - & - & - & - & - & 37 \\
\hline 14 & $2 \cdot 75$ & 3.08 & 3.43 & $3 \cdot 80$ & 4.25 & 4.78 & 5.64 & 708 & $\begin{array}{l}9.02 \\
6.002\end{array}$ & ang & - & - & - & - & $\boldsymbol{s \cdot 8}$ \\
\hline 29 & $2 \cdot 53$ & $2 \cdot 79$ & 307 & $3 \cdot 38$ & 3.70 & $4 \cdot 15$ & $4 \cdot 77$ & 562 & 6.89 & $8 \cdot 76$ & & & & - & $\mathbf{s \cdot 9}$ \\
\hline & $2 \cdot 38$ & 2.58 & $2 \cdot 82$ & 306 & 3.33 & 3.69 & $4 \cdot 14$ & $4 \cdot 77$ & $5 \cdot 50$ & 6.42 & $7 \cdot 40$ & 8.57 & $10 \cdot 12$ & & 40 \\
\hline 16 & $2 \cdot 31$ & $2 \cdot 47$ & $2 \cdot 65$ & $2 \cdot 85$ & 09 & 3.34 & $3 \cdot 72$ & $4 \cdot 15$ & $4 \cdot 61$ & $5 \cdot 14$ & 5.84 & 6.80 & $8 \cdot 44$ & 1100 & $4 \cdot 1$ \\
\hline & $2 \cdot 26$ & $2 \cdot 40$ & $2 \cdot 55$ & $2 \cdot 71$ & 2.88 & 3.10 & 3.37 & 3.67 & 4.03 & $4 \cdot 44$ & 5.04 & 5.94 & $7 \cdot 12$ & $8 \cdot 67$ & 42 \\
\hline & $2 \cdot 27$ & $2 \cdot 38$ & $2 \cdot 30$ & $2 \cdot 66$ & $2 \cdot \varepsilon$ & 2.96 & $3 \cdot 14$ & $3 \cdot 37$ & $3 \cdot 65$ & 4.01 & 4.35 & $5 \cdot 28$ & $6 \cdot 18$ & $7 \cdot 21$ & $4 \cdot 5$ \\
\hline & $2 \cdot 30$ & 2:41 & $2 \cdot 52$ & $2 \cdot 64$ & & 2.89 & 3.04 & 3.23 & $3 \cdot 48$ & $\mathbf{3} \cdot \mathbf{7 8}$ & 4.22 & 4.78 & 5.44 & 6.26 & 4.4 \\
\hline & $2 \cdot 35$ & $2 \cdot 44$ & 2.53 & 2.63 & $2 \cdot 75$ & 2.88 & 302 & $3 \cdot 20$ & $3 \cdot 40$ & $3 \cdot 65$ & 3-97 & $4 \cdot 40$ & $4-91$ & 5.56 & 4:5 \\
\hline & $2 \cdot 43$ & $2 \cdot 48$ & 2.56 & $2 \cdot 66$ & $2 \cdot 77$ & 2.89 & 3.01 & $3 \cdot 16$ & 3.34 & 3.55 & $3 \cdot \pi \theta$ & $4 \cdot 16$ & 4.55 & 5.10 & 46 \\
\hline & $2 \cdot 54$ & $2 \cdot 58$ & 263 & $2 \cdot 71$ & $2 \cdot 80$ & 2.90 & 301 & $3 \cdot 14$ & 3-29 & 3.47 & 3.67 & 3-96 & 4-28 & 4.72 & 47 \\
\hline $2 \cdot 70$ & $2 \cdot 72$ & 274 & $2 \cdot 78$ & 2.83 & & 2.97 & 3.08 & $3 \cdot 16$ & $3 \cdot 28$ & & 3.58 & 3.80 & 406 & $4 \cdot 42$ & $4 \cdot 8$ \\
\hline & $2 \cdot 97$ & $2 \cdot 96$ & $2 \cdot 97$ & 3.00 & & 3.08 & $3 \cdot 14$ & 3.21 & $3 \cdot 30$ & & 3.53 & 3.68 & 3.88 & $4 \cdot 16$ & 499 \\
\hline 3.31 & 3.25 & 3.21 & $3 \cdot 20$ & $3 \cdot 21$ & 3.22 & 3.24 & $3 \cdot 26$ & 3.31 & $3 \cdot 36$ & $3 \cdot 43$ & 3.51 & $3 \cdot 60$ & 373 & 3.96 & $\dot{b} \cdot 0$ \\
\hline & 3.64 & 3.55 & $3 \cdot 49$ & 3 & & 3.41 & 3.41 & 3.43 & 3.44 & $3 \cdot 46$ & 3.51 & 3.57 & & 378 & $5 \cdot 1$ \\
\hline & $4 \cdot 10$ & 3.8 & $3 \cdot 8$ & & & 3:63 & & 3.57 & & $3 \cdot 53$ & 3.53 & 3.55 & & $3 \cdot 67$ & $5 \cdot 2$ \\
\hline & 469 & $4 \cdot 4$ & $4 \cdot 2$ & & 4.02 & 3.92 & $3 \cdot 84$ & 3.76 & & $3 \cdot 63$ & $3 \cdot$ & 3.58 & & 3.62 & 5.8 \\
\hline & 3.44 & 30 & 4.80 & $4 \cdot 58$ & & 4.2 & $4 \cdot 13$ & 400 & 3.89 & 3:80 & 3.73 & $3 \cdot 68$ & 3.65 & 3.63 & 5.4 \\
\hline $6 \cdot 94$ & $6 \cdot 32$ & 5.82 & $5 \cdot 40$ & 6.07 & 4.84 & & $4 \cdot 46$ & $4 \cdot 30$ & & 4.06 & 3.95 & 3.87 & $3 \cdot 80$ & 3.75 & $5 \cdot 5$ \\
\hline- & - & 675 & 6.22 & 3.79 & 3.46 & $3 \cdot 19$ & 4.97 & 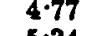 & 4.60 & $4 \cdot 44$ & $4 \cdot 30$ & $4 \cdot 18$ & $4 \cdot 10$ & 401 & $5 \cdot 6$ \\
\hline & - & $8 \cdot 15$ & $7 \cdot 30$ & 6.73 & 6.26 & $5 \cdot 91$ & $5 \cdot 61$ & 5.34 & $5 \cdot 10$ & 490 & $4 \cdot 73$ & $4 \cdot 59$ & $4: 47$ & $4: 38$ & 57 \\
\hline- & - & $10 \cdot 20$ & 8.76 & $7 \cdot 98$ & $7 \cdot 26$ & & & $5 \cdot 99$ & 5.68 & 5.44 & 5.24 & 5.06 & & 4.78 & 5.8 \\
\hline & - & 13.53 & $10 \cdot 83$ & $9 \cdot 68$ & & & & & 6.40 & & 5.84 & $5 \cdot 62$ & 5.44 & $5-28$ & $5 \cdot 9$ \\
\hline- & - & 19.96 & $14 \cdot 30$ & 1202 & 10.31 & 9:39 & & & $7 \cdot 39$ & & 6.61 & 6.33 & $6 \cdot 10$ & $5 \cdot 89$ & 60 \\
\hline & & & & & & 11.6 & 10.8 & $9 \cdot 31$ & $8:$ & $8 \cdot 03$ & $7 \cdot 53$ & $7 \cdot 15$ & & 6.54 & $6 \cdot 1$ \\
\hline & & & & & & & .55 & $11 \cdot 19$ & $10 \cdot 13$ & $9 \cdot 30$ & $8 \cdot 64$ & 8.08 & & 7.24 & 62 \\
\hline & & & - & & & $\mathbf{0}$ & 15.8 & $13 \cdot 69$ & e.21 & 1101 & 10.02 & $9 \cdot 19$ & & 801 & 6.3 \\
\hline & & & & & & & $20 \cdot 8$ & 17.09 & 14.56 & 12.8 & & 0.45 & $9 \cdot 60$ & $8 \cdot 92$ & 6.4 \\
\hline & - & - & - & & & 47.99 & 28.04 & $21 \cdot 30$ & $17 \cdot 44$ & 15.07 & $13 \cdot 20$ & 1.90 & $10-83$ & $10-07$ & 6.5 \\
\hline & - & - & - & - & - & - & - & & & & & & & & 6.6 \\
\hline & & & & & & & & & & & & $17^{\circ}$ & 15.8 & $14: 7$ & 67 \\
\hline & -7 & - & - & & & & & & - & 一 & - & $21 \cdot 8$ & $19 \cdot 6$ & $18 \cdot 3$ & 6.8 \\
\hline- & $\bar{z}$ & $=$ & $\bar{E}$ & - & $\bar{z}$ & $\pi$ & $\pi$ & - & - & $\bar{z}$ & Z & $29 \cdot 4$ & $\begin{array}{l}260^{\circ} \\
37.8\end{array}$ & & 699 \\
\hline & & & & & & & & - & & & & & & & \\
\hline
\end{tabular}

Biometrits II 
TABLE VI (i).

Values of $\boldsymbol{\beta}_{2}$.

\begin{tabular}{|c|c|c|c|c|c|c|c|c|c|c|c|}
\hline & $2 \cdot 0$ & $2 \cdot 5$ & 3.0 & 3.5 & 8.0 & $4: 5$ & $5 \cdot 0$ & 5.5 & 60 & 6.5 & $7 \cdot 0$ \\
\hline $\begin{array}{l}0 \cdot 0 \\
0 \cdot 1 \\
0 \cdot 2 \\
0 \cdot 3 \\
0 \cdot 4 \\
0.5 \\
0 \cdot 6 \\
0 \cdot 7 \\
0 \cdot 8 \\
0 \cdot 9 \\
1 \cdot 0 \\
1 \cdot 1 \\
1 \cdot 2 \\
1 \cdot 3 \\
1 \cdot 4 \\
1.5\end{array}$ & $\begin{array}{c}0 \\
0 \cdot 48493 \\
0 \cdot 91583 \\
1 \cdot 29873 \\
1.63902 \\
1.94118 \\
2 \cdot 20009 \\
2 \cdot 44615 \\
2 \cdot 65532 \\
2 \cdot 3917 \\
3 \cdot 00000 \\
3 \cdot 13980 \\
3 \cdot 26038 \\
3 \cdot 36330 \\
3 \cdot 45000 \\
3 \cdot 52174\end{array}$ & $\begin{array}{c}0 \\
0.68971 \\
1 \cdot 32958 \\
1 \cdot 86270 \\
2 \cdot 34286 \\
2 \cdot 78126 \\
3.17350 \\
3 \cdot 52441 \\
3 \cdot 83820 \\
4 \cdot 12064 \\
4 \cdot 36842 \\
4 \cdot 59081 \\
4 \cdot-78812 \\
4 \cdot 96250 \\
3 \cdot 11589 \\
5 \cdot 25000\end{array}$ & $\begin{array}{c}0 \\
0.94288 \\
1 \cdot 78182 \\
2 \cdot 53043 \\
3.20000 \\
3 \cdot-80000 \\
4.33846 \\
4.82228 \\
5.25714 \\
5 \cdot 64828 \\
6.00000 \\
6 \cdot 31613 \\
6.60000 \\
6 \cdot 85454 \\
7-08235 \\
7 \cdot 28571\end{array}$ & $\begin{array}{c}0 \\
1 \cdot 25688 \\
2 \cdot 37049 \\
3.36094 \\
4 \cdot 24478 \\
5.03582 \\
5.80178 \\
6 \cdot 38287 \\
6 \cdot 95698 \\
7 \cdot 47438 \\
7 \cdot 94121 \\
8 \cdot 36246 \\
8 \cdot 74286 \\
9.08619 \\
9 \cdot 39582 \\
9 \cdot 67501\end{array}$ & $\begin{array}{c}0 \\
1 \cdot 64906 \\
3 \cdot 10000 \\
4 \cdot 38305 \\
5 \cdot 528277 \\
6 \cdot 53846 \\
7 \cdot 4706 \\
8 \cdot 26202 \\
8 \cdot 99462 \\
9 \cdot 65454 \\
10 \cdot 24999 \\
10 \cdot 78796 \\
11 \cdot 27443 \\
11 \cdot 71461 \\
12 \cdot 1304 \\
12 \cdot 47368\end{array}$ & $\begin{array}{r}2 \cdot 14375 \\
4 \cdot 01176 \\
5 \cdot 65000 \\
7 \cdot 09474 \\
8 \cdot 37500 \\
9 \cdot 51429 \\
10 \cdot 53182 \\
11 \cdot 44375 \\
12 \cdot 26250 \\
13 \cdot 00000 \\
13 \cdot 66538 \\
14 \cdot 26667 \\
14 \cdot 81071 \\
15 \cdot 30345 \\
15 \cdot 75000\end{array}$ & \begin{tabular}{|}
$7 \cdot 2368$ \\
$9 \cdot 0462$ \\
10.6364 \\
12.0414 \\
$13 \cdot 2885$ \\
$14 \cdot 4000$ \\
$15 \cdot 3940$ \\
$16 \cdot 2857$ \\
17.0877 \\
$17 \cdot 8105$ \\
18.4633 \\
$19 \cdot 0536$ \\
$19 \cdot 5864$
\end{tabular} & $\mid \begin{array}{ll}15 & 1585 \\
16 \cdot 6624 \\
17 \cdot 9932 \\
19 \cdot 1758 \\
20 \cdot 2308 \\
21 \cdot-1750 \\
22.0225 \\
22 \cdot 7851 \\
23 \cdot 4727 \\
24.0937\end{array}$ & $\begin{array}{l} \\
23 \cdot 7791 \\
25.0000 \\
26.0857 \\
27.0546 \\
27.9217 \\
28 \cdot 7000 \\
29 \cdot 4000\end{array}$ & $\begin{array}{l}32 \cdot 0328 \\
33 \cdot 1082 \\
34 \cdot 0635 \\
34 \cdot 9164 \\
35 \cdot 6786\end{array}$ & $\begin{array}{l}42 \cdot 3613 \\
43 \cdot 1538\end{array}$ \\
\hline
\end{tabular}

TABLE VI (ii).

Values of $\beta_{4}$.

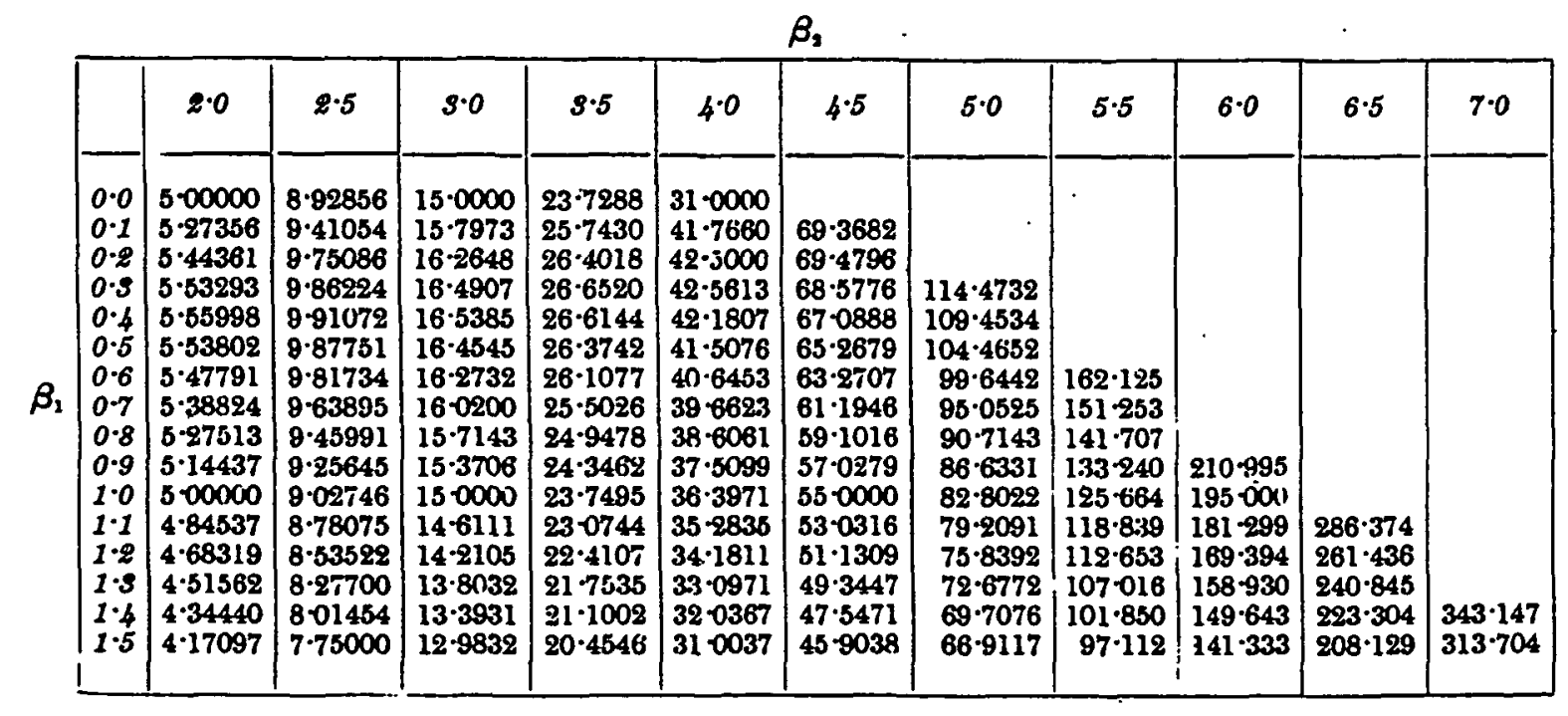


TABLE VI (iii).

Palues of $\beta_{1}$.

\begin{tabular}{|c|c|c|c|c|c|c|c|c|c|c|c|}
\hline & 20. & $2: 5$ & 50 & 3.5 & 40 & $4: 5$ & $5 \cdot 0$ & $5 \cdot 5$ & 6.0 & 6.5 & $7 \cdot 0$ \\
\hline $\begin{array}{l}0.0 \\
0.1 \\
0.2 \\
0.5 \\
0.4 \\
0.5 \\
0.6 \\
0.7 \\
0.8 \\
0.9 \\
1.0 \\
1.1 \\
1.2 \\
1.5 \\
1.4 \\
1.5\end{array}$ & $\begin{array}{c}0 \\
1.99086 \\
3.59438 \\
4.86677 \\
5 \cdot 85929 \\
6 \cdot 51704 \\
7 \cdot 17383 \\
7 \cdot 56616 \\
7 \cdot 81963 \\
7.95777 \\
8.00000 \\
7.98981 \\
7 \cdot 86015 \\
7 \cdot 70375 \\
7 \cdot 50358 \\
7.26808\end{array}$ & $\begin{array}{c}0 \\
4 \cdot 39480 \\
803374 \\
10 \cdot 68765 \\
18 \cdot 85477 \\
14 \cdot 51540 \\
15 \cdot 7892 \\
16 \cdot 8546 \\
17-2668 \\
176667 \\
17 \cdot 8291 \\
17 \cdot 8472 \\
17 \cdot 7503 \\
17 \cdot 5396 \\
17-2423 \\
16 \cdot 8768\end{array}$ & $\begin{array}{c}0 \\
9 \cdot 3207 \\
16 \cdot 5960 \\
22 \cdot 2196 \\
26-5187 \\
29 \cdot 7545 \\
32 \cdot 1362 \\
33-8306 \\
34-9714 \\
35-6658 \\
36-0000 \\
36-0437 \\
35-8535 \\
35 \cdot 4754 \\
34-9467 \\
34-2983\end{array}$ & 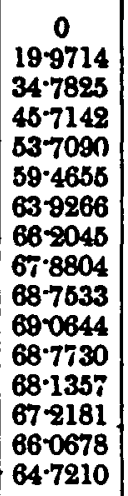 & 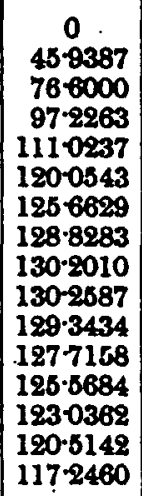 & $\begin{array}{l}128 \cdot 529 \\
193 \cdot 361 \\
228 \cdot 104 \\
246 \cdot 506 \\
255 \cdot 293 \\
258 \cdot 147 \\
257 \cdot 225 \\
253 \cdot 872 \\
248 \cdot 937 \\
243000 \\
236 \cdot 441 \\
229 \cdot 524 \\
222 \cdot 662 \\
214 \cdot 828 \\
207 \cdot 227\end{array}$ & \begin{tabular}{|l|}
$668 \cdot-284$ \\
$650 \cdot 398$ \\
614.633 \\
$381-205$ \\
$550 \cdot 107$ \\
521.257 \\
$495 \cdot 375$ \\
$469 \cdot 637$ \\
$446 \cdot 547$ \\
425.062 \\
$405 \cdot 663$ \\
$386 \cdot 347$ \\
$368 \cdot-843$
\end{tabular} & \begin{tabular}{|c|} 
\\
\\
$1618 \cdot 635$ \\
$1368 \cdot 373$ \\
$1196 \cdot 612$ \\
$1088 \cdot 877$ \\
$998 \cdot 318$ \\
$886 \cdot 341$ \\
$818 \cdot 040$ \\
$759 \cdot 48$ \\
$708 \cdot 620$ \\
$663 \cdot 926$
\end{tabular} & \begin{tabular}{|l}
$2769 \cdot 42$ \\
$2280 \cdot 00$ \\
$1945 \cdot 69$ \\
$1700 \cdot 98$ \\
1512.94 \\
$1363 \cdot 20$ \\
124063
\end{tabular} & $\begin{array}{l}3313 \cdot 80 \\
4135 \cdot 56 \\
3388 \cdot 18 \\
2870.08 \\
248862\end{array}$ & $\begin{array}{l}7265 \cdot 31 \\
5719 \cdot 68\end{array}$ \\
\hline
\end{tabular}

TABLE VI (iv).

Values of $\beta_{\text {s. }}$

\begin{tabular}{|c|c|c|c|c|c|c|c|c|c|c|c|}
\hline & $2 \cdot 0$ & 2.5 & 5.0 & 5.5 & 40 & $4: 5$ & $5 \cdot 0$ & 5.0 & 6.0 & 6.5 & $7 \cdot 0$ \\
\hline $\mid \begin{array}{l}0.0 \\
0 \cdot 1 \\
0.2 \\
0.5 \\
0.4 \\
0.5 \\
0.6 \\
0.7 \\
0.8 \\
0.9 \\
1.0 \\
1.1 \\
1.2 \\
1.5 \\
1.4 \\
1.5\end{array}$ & $\begin{array}{r}14 \cdot 0000 \\
16 \cdot 4616 \\
17.7286 \\
18 \cdot 1764 \\
18 \cdot 0667 \\
17.5474 \\
16 \cdot 8560 \\
15 \cdot 9787 \\
15.0148 \\
14.0113 \\
13.0000 \\
12 \cdot 0030 \\
11.0354 \\
10 \cdot 1070 \\
9 \cdot 2240 \\
8 \cdot 3899\end{array}$ & $\begin{array}{l}39-0649 \\
45 \cdot 7741 \\
50-2478 \\
51-0927 \\
50-2458 \\
487898 \\
46 \cdot 8558 \\
44 \cdot 3106 \\
41.7081 \\
39-0906 \\
38 \cdot 4119 \\
337916 \\
31.3418 \\
28.9775 \\
267355 \\
246268\end{array}$ & \begin{tabular}{|c|}
$105 \cdot 000$ \\
$124 \cdot 835$ \\
132.998 \\
$134-815$ \\
$131 \cdot 337$ \\
$126 \cdot 107$ \\
$119 \cdot 601$ \\
$112 \cdot 492$ \\
$105 \cdot 200$ \\
97.984 \\
91.000 \\
$84 \cdot 339$ \\
78.047 \\
$72 \cdot 146$ \\
$66 \cdot 637$ \\
$61 \cdot 512$
\end{tabular} & $\begin{array}{l}290 \cdot 678 \\
365 \cdot 508 \\
369 \cdot 894 \\
361 \cdot 909 \\
344 \cdot 886 \\
323.447 \\
303 \cdot 252 \\
277 \cdot 658 \\
255 \cdot 716 \\
235 \cdot 072 \\
216 \cdot 137 \\
198 \cdot-263 \\
181.987 \\
167 \cdot 142 \\
153 \cdot 682 \\
141 \cdot 477\end{array}$ & 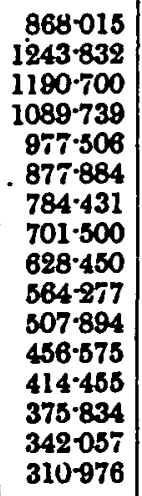 & $\begin{array}{r}10228 \cdot 33 \\
6204 \cdot 69 \\
4485 \cdot 38 \\
3471 \cdot 87 \\
2792 \cdot 19 \\
2303 \cdot 07 \\
193479 \\
1648 \cdot 52 \\
1420.51 \\
1235 \cdot 50 \\
1083-04 \\
955 \cdot 78 \\
848.97 \\
-75579 \\
676.32\end{array}$ & $\begin{array}{r}107697 \cdot 93 \\
25413 \cdot 18 \\
13737 \cdot 63 \\
9048 \cdot 43 \\
6534 \cdot 78 \\
5045 \cdot 80 \\
4024 \cdot 45 \\
3286 \cdot 65 \\
9741 \cdot 39 \\
9392 \cdot 13 \\
1994.03 \\
1796 \cdot 18 \\
1508 \cdot 92\end{array}$ & $\begin{array}{r}119230 \cdot 33 \\
411994 \cdot 77 \\
22660 \cdot 09 \\
14836 \cdot 90 \\
10618 \cdot 25 \\
8135 \cdot 91 \\
6314.06 \\
5108 \cdot 35 \\
4219 \cdot 50 \\
3544.82\end{array}$ & \begin{tabular}{|r|} 
\\
\\
$137288 \cdot 7$ \\
57584.9 \\
$33078 \cdot 5$ \\
$81891 \cdot 8$ \\
$15690 \cdot 2$ \\
11846.9 \\
$9281 \cdot 2$
\end{tabular} & $\begin{array}{r}797653 \cdot 2 \\
155693 \cdot 9 \\
75009 \cdot 7 \\
44891 \cdot 9 \\
30280 \cdot 3\end{array}$ & $\begin{array}{l}565740 \\
1857793\end{array}$ \\
\hline
\end{tabular}

$19=7$ 